Homology, Homotopy and Applications, vol.3, No.6, 2001, pp.111-138

\title{
SEMIDIRECT PRODUCTS OF CATEGORICAL GROUPS. OBSTRUCTION THEORY
}

\author{
ANTONIO R. GARZÓN AND HVEDRI INASSARIDZE \\ (communicated by Antonio Cegarra)
}

\begin{abstract}
By considering the notion of action of a categorical group $\mathbb{G}$ on another categorical group $\mathbb{H}$ we define the semidirect product $\mathbb{H} \ltimes \mathbb{G}$ and classify the set of all split extensions of $\mathbb{G}$ by $\mathbb{H}$. Then, in an analogous way to the group case, we develop an obstruction theory that allows the classification of all split extensions of categorical groups inducing a given pair $(\varphi, \psi)$ (called a collective character of $\mathbb{G}$ in $\mathbb{H})$ where $\varphi: \pi_{0}(\mathbb{G}) \rightarrow \pi_{0}(\mathcal{E} q(\mathbb{H}))$ is a group homomorphism and $\psi: \pi_{1}(\mathbb{G}) \rightarrow \pi_{1}(\mathcal{E} q(\mathbb{H}))$ is a homomorphism of $\pi_{0}(\mathbb{G})$-modules.
\end{abstract}

\section{Introduction}

An epimorphism of groups $\mu: E \longrightarrow G$ with kernel an abelian group $H$ induces, by conjugation in $E$, a $G$-module structure in $H$ or, equivalently, a group homomorphism $p: G \rightarrow A u t(H)$. The problem of classifying, up to equivalence, all such extensions of $G$ by $H$ which induce $p$ was solved in the 1930's by using factor sets $[\mathbf{1}],[\mathbf{1 8}]$, and later $[\mathbf{1 1}]$ by means of the 2-dimensional cohomology group of $G$ with coefficients in $H$.

If the group $H$ is non-abelian, for any normalized set-theoretical section of $\mu$, there is a pair of maps $(f: G \times G \rightarrow H, \alpha: G \rightarrow A u t(H))$ satisfying certain conditions, which is called a factor set (or 2-cocycle) of $G$ with coeffcients in $H$. This factor set determines, up to equivalence, the extension and in this way Schreier's theorem [18] of classification of extensions of groups with non-abelian kernel assures the existence of a natural bijection

$$
\mathcal{H}^{2}(G, H) \cong \operatorname{Ext}(G, H)
$$

between the set of equivalence classes of factor sets of $G$ by $H$ and the set of equivalence classes of extensions of $G$ by $H$. In particular, let us remark that, by this bijection, split extensions correspond to the classes of factor sets of the form $(0, \alpha)$ with $\alpha: G \rightarrow \operatorname{Aut}(H)$ a group homomorphism ( classification of semidirect product extensions).

This paper has been financially supported by NATO PST.CLG 975316.

Received October 31, 2000, revised April 24, 2001; published on May 8, 2001.

2000 Mathematics Subject Classification: 18D10, 18G50.

Key words and phrases: Categorical group, semidirect product, split extension, obstruction.

(c) 2001, Antonio R. Garzón and Hvedri Inassaridze. Permission to copy for private use granted. 
In $\S 3$ of this paper we give the corresponding version, of the theorem of classification of split extensions of groups, in the more general setting of categorical groups. The analysis of the developing, in the last decades, of results extending the classical results on group extensions, their cohomological classification and their topological interpretation (c.f. $[\mathbf{9}],[\mathbf{1 0}],[\mathbf{8}]$ ) reveals that the key of such a developing lies in the consideration of (internal) groupoids instead of groups. In this sense, the 2-category of the categorical groups has joined (see $[\mathbf{1 9}],[\mathbf{3}],[\mathbf{2 1}],[\mathbf{6}],[\mathbf{7}])$ as an adequate context in which to approach problems of the same nature as the above-mentioned but from a more general point of view, thus obtaining a wider range of applications. For instance, the classification of split extensions of categorical groups is the algebraization of a topological problem consisting of the classification of those fibrations of spaces $p: E \rightarrow B$ which admit a cross-section, where both the base space $B$ and the fiber of $p, F$, have the homotopy type of a categorical group [19].

In $\S 2$ we start by giving a summary of terminology, some significant examples and recalling some known results of categorical groups. Then, in $\S 3$ we make the transition to this context of main notions in group theory such as those of action of a categorical group on another one, of the categorical group semidirect product and the corresponding semidirect product extension. With the suitable notion of equivalence, both for actions and extensions, we conclude in Theorem 3.9 the classification of all split extensions of categorical groups. Using these notions, we expect to develop in a forthcoming paper a study of derivations (or crossed homomorphisms) from a categorical group $\mathbb{G}$ to a $\mathbb{G}$-categorical group $\mathbb{H}$ and its relation with cohomology in this context.

Coming back again to the classical precedents, let us recall that, in the setting of the group extensions theory, there exists (c.f. $[\mathbf{1}],[\mathbf{1 1}],[\mathbf{9}]$ ) an "obstruction problem" consisting of the classification of all group extensions $1 \rightarrow H \rightarrow E \rightarrow G \rightarrow 1$ which induce a given group homomorphism $p: G \rightarrow \operatorname{Out}(H)$ where $\operatorname{Out}(H)=$ $\frac{\operatorname{Aut}(H)}{\operatorname{Int}(H)}$. Each extension certainly induces a homomorphism $G \rightarrow O u t(H)$ but such a homomorphism $p$ need not always be induced by an extension. The existence of an extension inducing $p$ is measured by a 3-cocycle of $G$ with coefficients in the $G$-module center of $H$, which is called the obstruction of $p$. Note that if $1 \rightarrow$ $H \rightarrow E \stackrel{\mu}{\rightarrow} G \rightarrow 1$ is a split extension (i.e., there is a group homomorphism $s$ : $G \rightarrow E$ such that $\left.\mu s=i d_{G}\right)$ and $\nu: E \rightarrow A u t(H)$ is the homomorphism given by conjugation in $E$, then there is a homomorphism $\nu s: G \rightarrow \operatorname{Aut}(H)$. Thus the problem in this case consists of measuring the obstruction of those homomorphisms $p: G \rightarrow \operatorname{Out}(H)$ such that $p=q \alpha$ where $\alpha: G \rightarrow \operatorname{Aut}(H)$ and $q: \operatorname{Aut}(H) \rightarrow$ $\operatorname{Out}(H)$ is the projection. In this way, the obstruction theorems for group extensions were formulated, providing on the other way and during years, the only known interpretation of the third cohomology group of $G$ with coefficients in a $G$-module A.

In $\S 4$ we approach, in the context of the theory of extensions of categorical groups, an analogous problem of obstruction in the case of considering split extensions of a categorical group $\mathbb{G}$ by a categorical group $\mathbb{H}$. Each split extension

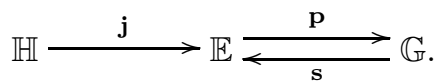


induces what we call a collective character of $\mathbb{G}$ in $\mathbb{H}$, that is, a pair $(\varphi, \psi)$ where $\varphi: \pi_{0}(\mathbb{G}) \rightarrow \pi_{0}(\mathcal{E} q(\mathbb{H}))$ is a group homomorphism and $\psi: \pi_{1}(\mathbb{G}) \rightarrow \pi_{1}(\mathcal{E} q(\mathbb{H}))$ is a homomorphism of $\pi_{0}(\mathbb{G})$-modules. However, such a pair $(\varphi, \psi)$ need not always be induced (realized) by a split extension of $\mathbb{G}$ by $\mathbb{H}$ and its existence can be measured by a 3 -cocycle of $\pi_{0}(\mathbb{G})$ with coefficients in $\pi_{1}(\mathcal{E} q(\mathbb{H}))$ which is called the obstruction of the collective character $(\varphi, \psi)$. We then conclude this section by formulating in 4.3 and 4.4 the corresponding obstruction theorems:

" A collective character $(\varphi, \psi)$ of a categorical group $\mathbb{G}$ in a categorical group $\mathbb{H}$ is realizable if and only if its obstruction $k(\varphi, \psi) \in H_{\varphi}^{3}\left(\pi_{0}(\mathbb{G}), \pi_{1}(\mathcal{E} q(\mathbb{H}))\right)$ vanishes",

"If a collective character $(\varphi, \psi)$ is realizable then the set $\mathbf{E x t}_{\text {split }}^{(\varphi, \psi)}(\mathbb{G}, \mathbb{H})$ of equivalence classes of realizations of $(\varphi, \psi)$ is a principal homogeneous space under the abelian group $H_{\varphi}^{2}\left(\pi_{0}(\mathbb{G}), \pi_{1}(\mathcal{E} q(\mathbb{H}))\right)$. In particular, there is a (non-natural) bijection

$$
\operatorname{Ext}_{\text {split }}^{(\varphi, \psi)}(\mathbb{G}, \mathbb{H}) \cong H_{\varphi}^{2}\left(\pi_{0}(\mathbb{G}), \pi_{1}(\mathcal{E} q(\mathbb{H}))\right) .
$$

\section{Categorical groups: Notation and preliminary results}

In this preliminary section we recall the main definitions and first results concerning the 2-category of categorical groups. We refer to $[\mathbf{1 5}],[\mathbf{1 7}],[\mathbf{2}],[\mathbf{3}],[\mathbf{1 4}]$, [13], [19] for general background about them.

A monoidal category $\mathbb{G}=(\mathbb{G}, \otimes, a, I, l, r)$ consists of a category $\mathbb{G}$, a functor (tensor product) $\otimes: \mathbb{G} \times \mathbb{G} \rightarrow \mathbb{G}$, an object $I$ (unit) and natural isomorphisms called, respectively, the associativity, left unit and right unit constraints

$$
\begin{gathered}
a=a_{X, Y, Z}:(X \otimes Y) \otimes Z \stackrel{\sim}{\longrightarrow} X \otimes(Y \otimes Z) \\
l=l_{X}: I \otimes X \stackrel{\sim}{\longrightarrow} X \quad, \quad r=r_{X}: X \otimes I \stackrel{\sim}{\longrightarrow} X,
\end{gathered}
$$

such that for any objects $X, Y, Z, W \in \mathbb{G}$ the following diagrams (associativity coherence and unit coherence) are commutative:

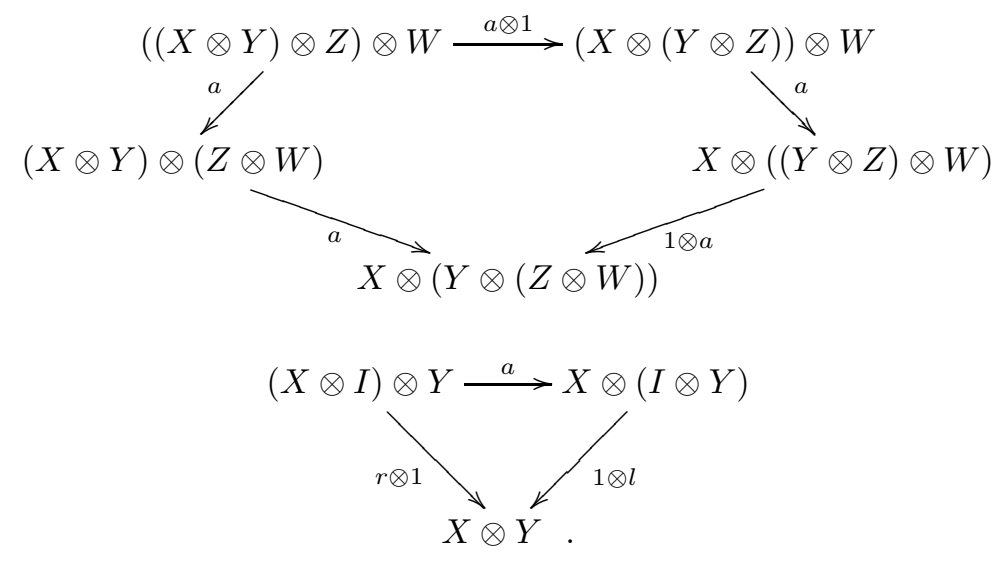


Let us not that the naturality of $l$ and $r$ imply that, for any $X \in \mathbb{G}$, the following equalities are satisfied:

$$
l_{I \otimes X}=1_{I} \otimes l_{X} \quad ; \quad r_{X \otimes I}=r_{X} \otimes 1_{I},
$$

and from the commutativity of (2) we have that

$$
r_{I}=l_{I}: I \otimes I \stackrel{\sim}{\longrightarrow} I,
$$

and also the commutativity of the following diagrams:
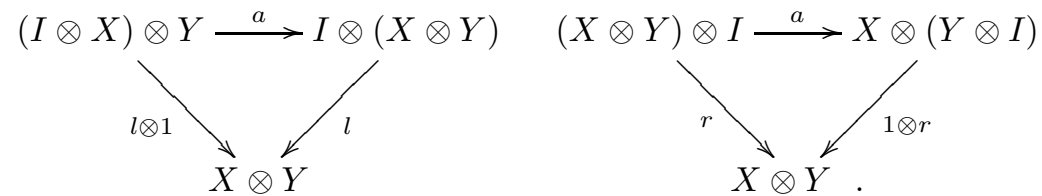

In a monoidal category, an object $X$ is termed invertible if the functors $Y \mapsto$ $X \otimes Y$ and $Y \mapsto Y \otimes X$ are equivalences. The natural isomorphisms of left and right unit say just that the unit $I$ is an invertible object.

A (right) inverse for an invertible object $\mathrm{X}$ consists of an object $X^{*}$ and an isomorphism $\alpha_{X}: X \otimes X^{*} \stackrel{\sim}{\rightarrow} I$ (this isomorphism exists since $X \otimes-$ is an autoequivalence). For other choice of inverse $\left(X^{0}, \alpha_{X}^{\prime}\right)$ there exists an isomorphism $\varphi: X^{*} \stackrel{\sim}{\rightarrow} X^{0}$ determined uniquely by the commutativity of the diagram

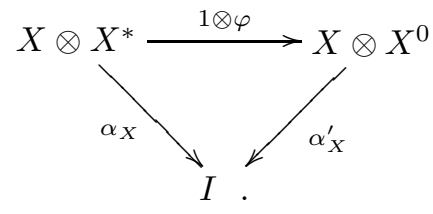

A categorical group $\mathbb{G}$ is a monoidal small category where every object is invertible and every morphism is an isomorphism (i.e., $\mathbb{G}$ is a groupoid). A categorical group is termed strict when the isomorphisms of associativity and left and right unit are identity arrows and the isomorphisms $\alpha_{X}: X \otimes X^{*} \stackrel{\sim}{\rightarrow} I$ can be chosen as identities. Any categorical group is equivalent to a strict one. This fact, commonly assumed in the literature, can be obtained for instance as a consequence of Proposition 1.5 and Theorem 2.6 in [5].

In a categorical group $\mathbb{G}$, once a system of (right) inverses $\left(X^{*}, \alpha_{X}\right)$ has been chosen, the isomorphisms $\alpha_{X}$ determine isomorphisms $\beta_{X}: X^{*} \otimes X \stackrel{\sim}{\rightarrow} I$ by means of the following commutative diagram:

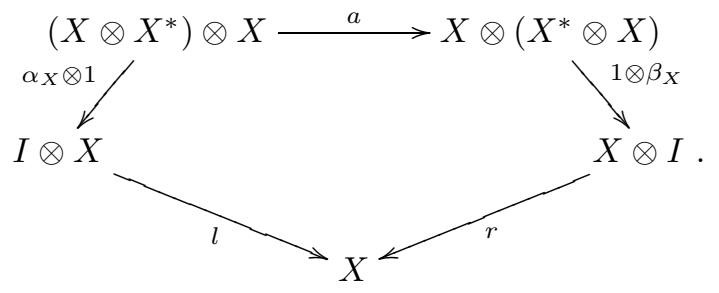


The isomorphisms $\beta$ come to say that $X^{*}$ is also a left inverse for $X$ and, actually, the isomorphisms $\alpha_{X}$ and $\beta_{X}$ determine each other and $\left(X, X^{*}, \alpha_{X}, \beta_{X}\right)$ is a duality in $\mathbb{G}$. The choice, for each $X$, of such a duality induces a contravariant endofunctor of $\mathbb{G}, X \mapsto X^{*},(X \stackrel{f}{\rightarrow} Y) \mapsto\left(Y^{*} \stackrel{f^{*}}{\rightarrow} X^{*}\right)$ (where $f^{*}=l_{X^{*}}\left(\beta_{Y} \otimes 1\right) a_{Y^{*}, Y, X}^{-1}(1 \otimes f \otimes$ $\left.1)\left(1 \otimes \alpha_{X}^{-1}\right) r_{Y^{*}}^{-1}\right)$, such that the isomorphisms $\alpha_{X}$ and $\beta_{X}$ are natural.

Let us remark that, once a system of inverses has been chosen, there are natural isomorphisms:

$$
X \stackrel{\sim}{\longrightarrow}\left(X^{*}\right)^{*} ;(X \otimes Y)^{*} \stackrel{\sim}{\longrightarrow} Y^{*} \otimes X^{*} .
$$

Suppose now that $\mathbb{G}$ and $\mathbb{H}$ are categorical groups. A homomorphism $\mathbf{T}=(T, \mu)$ : $\mathbb{G} \rightarrow \mathbb{H}$ consists of a functor $T: \mathbb{G} \rightarrow \mathbb{H}$ and a family of natural isomorphisms

$$
\mu=\mu_{X, Y}: T(X \otimes Y) \longrightarrow T(X) \otimes T(Y),
$$

such that, for any objects $X, Y, Z \in \mathbb{G}$, the following diagram is commutative:

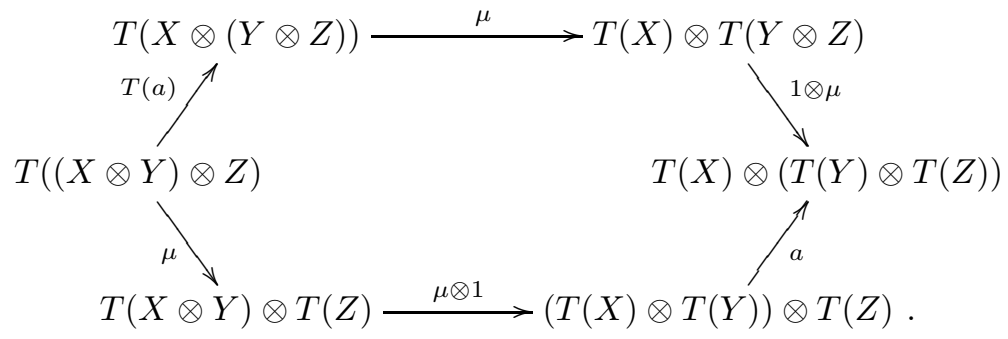

If $T: \mathbb{G} \rightarrow \mathbb{H}$ is a homomorphism, there exists an isomorphism,

$$
\mu_{0}: T(I) \stackrel{\sim}{\longrightarrow} I,
$$

determined uniquely by the commutativity of the two following diagrams:

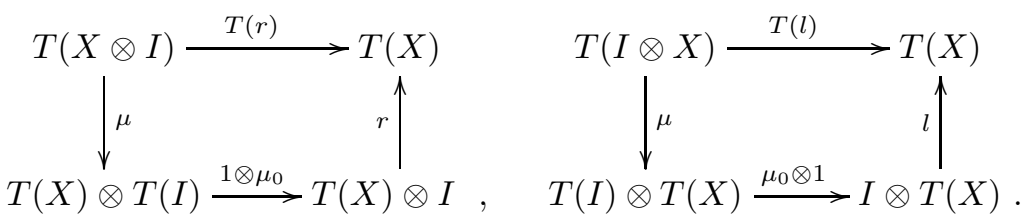

The homomorphism $(T, \mu)$ is called strict when each of the isomorphisms $\mu_{X, Y}$ and $\mu_{0}$ is an identity.

Let us note that, once a system of inverses $\left(X^{*}, \alpha_{X}\right)$ for $X \in \mathbb{G}$ and $\left(Y^{*}, \alpha_{Y}\right)$ for $Y \in \mathbb{H}$ has been chosen, there exist unique isomorphisms

$$
\lambda_{X}: T\left(X^{*}\right) \stackrel{\sim}{\longrightarrow} T(X)^{*}
$$

such that the following diagrams commute: 


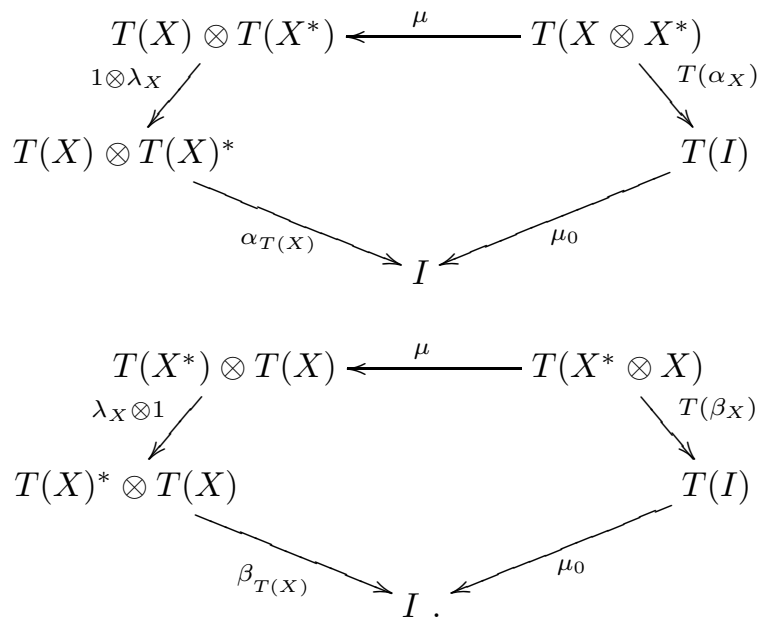

If $(T, \mu): \mathbb{G} \rightarrow \mathbb{H}$ and $\left(T^{\prime}, \mu^{\prime}\right): \mathbb{H} \rightarrow \mathbb{K}$ are homomorphisms of categorical groups, their composite is defined by $\left(T^{\prime \prime}, \mu^{\prime \prime}\right): \mathbb{G} \rightarrow \mathbb{K}$, where $T^{\prime \prime}=T^{\prime} T: \mathbb{G} \rightarrow \mathbb{K}$ and $\mu^{\prime \prime}=\mu_{X, Y}^{\prime \prime}: T^{\prime} T(X \otimes Y) \rightarrow T^{\prime} T(X) \otimes T^{\prime} T(Y)$ is the isomorphism given by the composition $\mu_{T X, T Y}^{\prime} T^{\prime}\left(\mu_{X, Y}\right)$.

Given homomorphisms of categorical groups $(T, \mu),\left(T^{\prime} \mu^{\prime}\right): \mathbb{G} \rightarrow \mathbb{H}$, a morphism from $(T, \mu)$ to $\left(T^{\prime}, \mu^{\prime}\right)$ consists of a natural transformation $\epsilon: T \rightarrow T^{\prime}$ such that, for any objects $X, Y \in \mathbb{G}$, the following diagram is commutative:

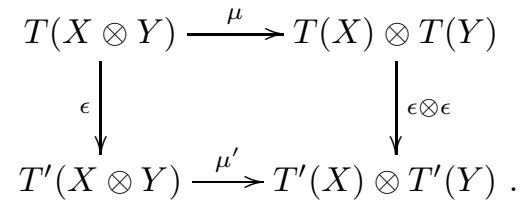

Observe that a natural transformation between two homomorphisms of categorical groups is necessarily a natural isomorphism.

If $\epsilon:(T, \mu) \rightarrow\left(T^{\prime}, \mu^{\prime}\right)$ is a morphism, then the following diagrams are commutative:
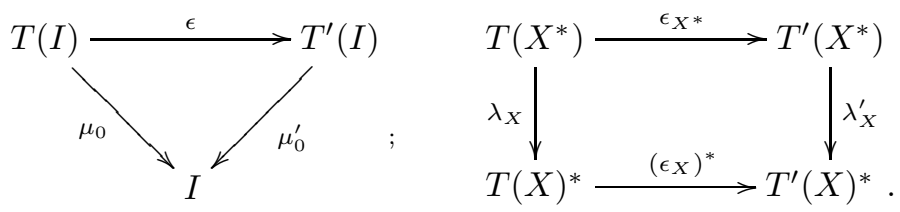

All the above considerations yield to the 2-category of categorical groups, whose 1-cells are the homomorphisms of categorical groups and whose 2-cells are the morphisms between them. 


\section{Examples}

1. If $G$ is a group, the discrete category it defines, denoted by $G \rightrightarrows G$, is a strict categorical group where the tensor product is given by the group operation. In the case that $G$ is an abelian group, then the category with only one object it defines, denoted by $G \rightrightarrows 1$, is also a strict categorical group where both the composition and the tensor product are given by the group operation.

2. It is well known that strict categorical groups or, equivalently, groupoids in the category of groups, are the same as Whitehead crossed modules, [4]. Recall that a crossed module of groups is a system $\Phi=(H, \pi, \varphi, \rho)$, where $\rho: H \rightarrow \pi$ is a group homomorphism and $\varphi: \pi \rightarrow \operatorname{Aut}(H)$ is an action (so that $H$ is a $\pi$-group) for which the following conditions are satisfied:

$$
\rho\left({ }^{a} h\right)=a \rho(h) a^{-1} \quad ; \quad \rho(h) h^{\prime}=h h^{\prime} h^{-1} .
$$

Given a crossed module $\Phi$, the corresponding strict categorical group $\mathbb{G}(\Phi)$ can be described as follows: The objects are the elements of the group $\pi$; an arrow $h: a \rightarrow b$ is an element $h \in H$ with $a=\rho(h) b$. The composition is multiplication in $H$. The tensor product is given by

$$
(a \stackrel{h}{\longrightarrow} b) \otimes\left(c \stackrel{h^{\prime}}{\longrightarrow} d\right)=\left(a c \stackrel{h^{b} h^{\prime}}{\longrightarrow} b d\right) .
$$

3. Suppose $T: \mathbb{G} \rightarrow \mathbb{H}$ is an equivalence of groupoids. Then each categorical group structure on $\mathbb{G}$ transports along $T$ to a categorical group structure on $\mathbb{H}$. This in particular applies to the case when $\mathbb{G}$ is a strict categorical group and in this way one can obtain many algebraic examples of categorical groups.

4. The categorical group of loops of a pointed space, $\mathcal{P}_{2}(X, *)$ (see [12]).

Let us denote by $\mathcal{P}_{1}(Y)$ the fundamental groupoid of a topological space $Y$. If $(X, *)$ is a pointed topological space with base point $* \in X$, then $\mathcal{P}_{2}(X, *)=$ $\mathcal{P}_{1}(\Omega(X, *))$, that is, the fundamental groupoid of the loop space $\Omega(X, *)$. Thus, the objects are the maps $\omega: I \rightarrow X$ such that $\omega(0)=*=\omega(1)$, and the morphisms $[f]: \omega \rightarrow \omega^{\prime}$ are homotopy classes rel end loops of homotopies $f: \omega \rightarrow \omega^{\prime}$ rel end points. The composition of two morphisms in $\mathcal{P}_{2}(X, *)$, $[f]: \omega \rightarrow \omega^{\prime}$ and $[g]: \omega^{\prime} \rightarrow \omega^{\prime \prime}$ is defined by $[g][f]=[g f]$, where $g f: I \times I \rightarrow X$ is the map $(g f)(t, s)=\left\{\begin{array}{ll}f(t, 2 s) & 0 \leqslant t \leqslant 1 / 2 \\ g(t, 2 s-1) & 1 / 2 \leqslant s \leqslant 1\end{array}\right.$.

Since the functor $\mathcal{P}_{1}$ preserves products, the map $\mu: \Omega(X, *) \times \Omega(X, *) \rightarrow$ $\Omega(X, *)$ defined by

$$
\mu\left(\omega, \omega^{\prime}\right)= \begin{cases}\omega(2 t) & 0 \leqslant t \leqslant 1 / 2 \\ \omega^{\prime}(2 t-1) & 1 / 2 \leqslant t \leqslant 1\end{cases}
$$

induces a functor $\otimes: \mathcal{P}_{2}(X, *) \times \mathcal{P}_{2}(X, *) \rightarrow \mathcal{P}_{2}(X, *)$ that is given on objects by $\omega \otimes \omega^{\prime}=\mu\left(\omega, \omega^{\prime}\right)$ and, on morphisms, by $[f] \otimes[g]=[f \otimes g]$ where $(f \otimes g)(t, s)=\left\{\begin{array}{ll}f(2 t, s) & 0 \leqslant t \leqslant 1 / 2 \\ g(2 t-1, s) & 1 / 2 \leqslant t \leqslant 1\end{array}\right.$.

There is an associativity constraint $a:\left(\omega \otimes \omega^{\prime}\right) \otimes \omega^{\prime \prime} \rightarrow \omega \otimes\left(\omega^{\prime} \otimes \omega^{\prime \prime}\right)$ which 
is defined as the homotopy class of the map $A: I \times I \rightarrow X$ given by

$$
A(s, t)= \begin{cases}\omega\left(\frac{4 s}{t+1}\right) & 0 \leqslant s \leqslant \frac{t+1}{4} \\ \omega^{\prime}(4 s-t-1) & \frac{t+1}{4} \leqslant s \leqslant \frac{t+2}{4} \\ \omega^{\prime \prime}\left(\frac{4 s-2-t}{2-v}\right) & \frac{t+2}{4} \leqslant s \leqslant 1\end{cases}
$$

and there are a unit object $*$, which is the constant map from $I$ to $*$, and unit constraints

$$
l=[L]: * \otimes \omega \rightarrow \omega, \quad r=[R]: \omega \otimes * \rightarrow \omega,
$$

where $L, R: I \times I \rightarrow X$ are respectively defined by

$$
L(s, t)= \begin{cases}* & 0 \leqslant s \leqslant \frac{1-t}{2} \\ \omega\left(\frac{2 s+t-1}{1+t}\right) & \frac{1-t}{2} \leqslant s \leqslant 1\end{cases}
$$

and

$$
R(s, t)=\left\{\begin{array}{ll}
\omega\left(\frac{2 s}{t+1}\right) & 0 \leqslant s \leqslant \frac{t+1}{2} \\
* & \frac{t+1}{2} \leqslant s \leqslant 1
\end{array},\right.
$$

such that $\mathcal{P}_{2}(X, *)=\left(\mathcal{P}_{2}(X), \otimes, a, *, l, r\right)$ is a categorical group.

5. The categorical group $\mathcal{E} q(\mathbb{G}, c)$ of the equivalences of a categorical group.

An equivalence of a categorical group $\mathbb{G}$ is a homomorphism $(T, \mu): \mathbb{G} \rightarrow \mathbb{G}$ such that the endofunctor $T: \mathbb{G} \rightarrow \mathbb{G}$ is an equivalence of categories. The objects of $\mathcal{E} q(\mathbb{G})$ are the equivalences of $\mathbb{G}$ and the arrows are the morphisms between them. The composition in $\mathcal{E} q(\mathbb{G})$ is given by the usual vertical composition of natural transformations, $\left(\epsilon^{\prime} \cdot \epsilon\right)_{X}=\epsilon_{X}^{\prime} \epsilon_{X}$, which is again a morphism in $\mathcal{E} q(\mathbb{G})$. It is clear that $\mathcal{E} q(\mathbb{G})$ is a groupoid. The composition of the homomorphisms and the horizontal composition of the natural transformations define a tensor functor $\otimes: \mathcal{E} q(\mathbb{G}) \times \mathcal{E} q(\mathbb{G}) \rightarrow \mathcal{E} q(\mathbb{G})$, that is, given $\epsilon:(T, \mu) \Rightarrow\left(T^{\prime}, \mu^{\prime}\right)$ and $\epsilon^{\prime}:\left(T^{\prime \prime}, \mu^{\prime \prime}\right) \Rightarrow\left(T^{\prime \prime \prime}, \mu^{\prime \prime \prime}\right)$, then $\epsilon^{\prime} \otimes \epsilon$ is defined by $\left(\epsilon^{\prime} \otimes \epsilon\right)_{X}=\epsilon_{T^{\prime} X}^{\prime} T^{\prime \prime}\left(\epsilon_{X}\right)=T^{\prime \prime \prime}\left(\epsilon_{X}\right) \epsilon_{T X}^{\prime}$. Thus, $\mathcal{E} q(\mathbb{G})$ is a categorical group in which $I=1_{\mathbb{G}}$ and an inverse for an object $(T, \mu)$ is obtained by taking a quasiinverse of $T$.

Let us remark that $\mathcal{E} q(\mathbb{G})$ has a categorical subgroup, $\mathcal{A} u t(\mathbb{G})$, whose objects, called automorphisms, are the equivalences $(T, \mu)$ that are strict and where $T$ is an isomorphism.

Suppose that $\mathbb{G}$ is a categorical group. Then, the set of connected components of $\mathbb{G}, \pi_{0}(\mathbb{G})$, has a group structure where the operation is given by $[X] \cdot[Y]=$ $[X \otimes Y]$. This is a well defined operation as consequence of the functoriality of $\otimes$; the associativity is consequence of the associativity constraints and the unit constraints assure that $[I]$ is a neutral element; finally, since the translations are autoequivalences, every element has an inverse. On the other hand, $\mathbb{G}$ has also associated the abelian group $\pi_{1}(\mathbb{G})=A u t_{\mathbb{G}}(I)$. For instance, the group of connected components of $\mathcal{P}_{2}(X, *)$ is $\pi_{1}(X, *)$ and the group of automorphisms in the unit object is $\pi_{2}(X, *)$.

For every object $X \in \mathbb{G}$ there are maps

$$
\gamma_{X}, \delta_{X}: \pi_{1}(\mathbb{G}) \rightarrow \operatorname{Aut}_{\mathbb{G}}(X)
$$


which are defined, for each $u \in \pi_{1}(\mathbb{G})$, as the unique morphisms making commutative the following diagrams:
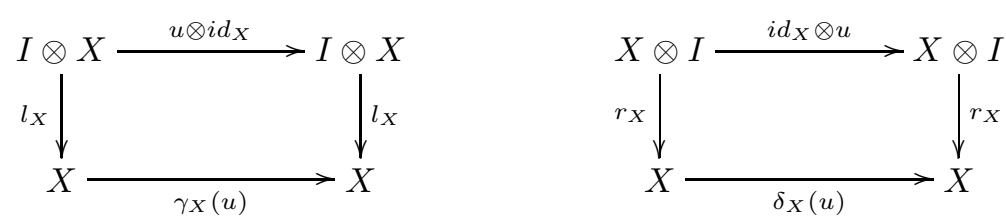

that is,

$$
\gamma_{X}(u)=l_{X}\left(u \otimes i d_{X}\right) l_{X}^{-1} \quad, \quad \delta_{X}(u)=r_{X}\left(i d_{X} \otimes u\right) \delta_{X}^{-1} .
$$

These maps $\gamma_{X}$ and $\delta_{X}$ are group isomorphisms [19] and then, for any object $X \in \mathbb{G}$, the group $A u t_{\mathbb{G}}(X)$ is abelian.

These isomorphisms are compatible with the functor $\otimes$ in the sense that, for any objects $X, Y \in \mathbb{G}$ and any $u \in \pi_{1}(\mathbb{G})$, the following equalities are satisfied:

$$
\gamma_{X \otimes Y}(u)=\gamma_{X}(u) \otimes i d_{Y} ; \delta_{X \otimes Y}(u)=i d_{X} \otimes \delta_{Y}(u) ; \delta_{X}(u) \otimes i d_{Y}=i d_{X} \otimes \gamma_{Y}(u) \text {. (9) }
$$

Moreover, if $f: X \rightarrow Y$ is a morphism in $\mathbb{G}$, the following diagrams are commutative
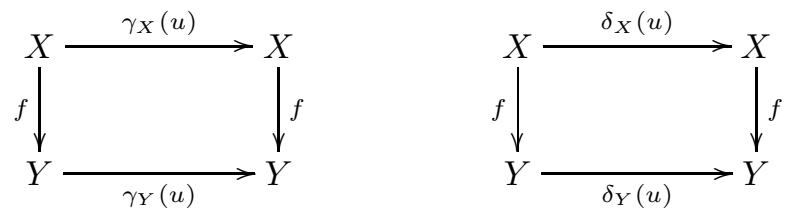

and then, the following diagram is also commutative

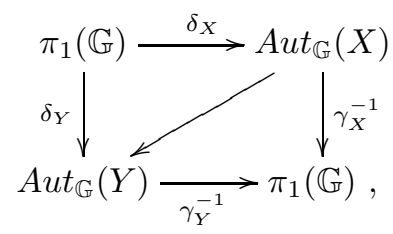

where the diagonal morphism is given by $g \mapsto f g f^{-1}$. All these facts allow to show [19] that for any two objects $X, Y \in \mathbb{G}$ and morphisms $f, g: X \rightarrow Y$ there exists a unique element $u \in \pi_{1}(\mathbb{G})$ such that the following equality is satisfied

$$
g=f \gamma_{X}(u)=\gamma_{Y}(u) f,
$$

and therefore there is a map

$$
\pi_{0}(\mathbb{G}) \times \pi_{1}(\mathbb{G}) \rightarrow \pi_{1}(\mathbb{G}), \quad([X], u) \mapsto \gamma_{X}^{-1} \delta_{X}(u),
$$

giving to $\pi_{1}(\mathbb{G})$ a $\pi_{0}(\mathbb{G})$-module structure.

Let us remark that, for any objects $X, Y \in \mathbb{G}$ and any $v \in \pi_{1}(\mathbb{G})$, the following equalities are satisfied

$$
\begin{gathered}
i d_{X} \otimes \gamma_{Y}(v)=\delta_{X}(v) \otimes i d_{Y}= \\
=\gamma_{X} \gamma_{X}^{-1} \delta_{X}(v) \otimes i d_{Y}=\gamma_{X \otimes Y}\left(\gamma_{X}^{-1} \delta_{X}(v)\right)=\gamma_{X \otimes Y}\left[{ }^{[X]} v\right)
\end{gathered}
$$


and therefore, for any $u, v \in \pi_{1}(\mathbb{G})$, we have:

$$
\begin{gathered}
\gamma_{X}(u) \otimes \gamma_{Y}(v)=\left(\gamma_{X}(u) \otimes i d_{Y}\right)\left(i d_{X} \otimes \gamma_{Y}(v)\right)= \\
=\gamma_{X \otimes Y}(u) \gamma_{X \otimes Y}\left({ }^{[X]} v\right)=\gamma_{X \otimes Y}\left(u^{[X]} v\right) .
\end{gathered}
$$

If for each $x \in \pi_{0}(\mathbb{G})$ we choose a representative $X_{x} \in x$ with $X_{1}=I$, where $1=[I]$, and for any $Y \in x$ we choose a morphism $i_{Y}: Y \rightarrow X_{x}$ such that $i_{X_{x}}=i d_{X_{x}}$, $i_{I \otimes X_{x}}=l_{X_{x}}$ and $i_{X_{x} \otimes I}=r_{X_{x}}$, then, for any three elements $x, y, z \in \pi_{0}(\mathbb{G})$, we can consider the following automorphism of $X_{x y z}$ :

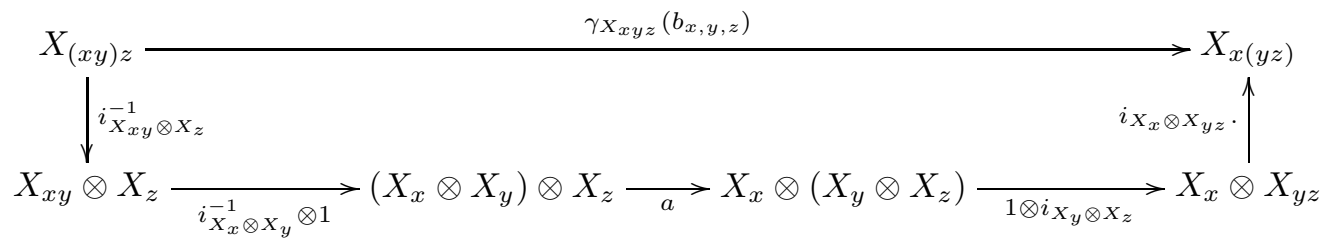

Thus, as consequence of the coherence of the associativity constraint (1), the element $b_{x, y, z} \in \pi_{1}(\mathbb{G})$ determines a normalized 3-cocycle $b$ of $\pi_{0}(\mathbb{G})$ with coefficients in the $\pi_{0}(\mathbb{G})$-module $\pi_{1}(\mathbb{G})$. Moreover, for any other choices of objects $\bar{X}_{x} \in x$ and of morphisms $j_{Y}: Y \rightarrow \bar{X}_{x}$, the new 3-cocycle $\bar{b}$ is cohomologous to $b$ (see [19]).

Finally, note that if $\mathbb{G}$ and $\mathbb{H}$ are categorical groups and $\mathbf{T}=(T, \mu): \mathbb{G} \rightarrow \mathbb{H}$ is a homomorphism of categorical groups, then $T$ induces a group homomorphism $\pi_{0}(T): \pi_{0}(\mathbb{G}) \rightarrow \pi_{0}(\mathbb{H}),[X] \mapsto[T X]$, and a homomorphism of $\pi_{0}(\mathbb{G})$-modules $\pi_{1}(T): \pi_{1}(\mathbb{G}) \rightarrow \pi_{1}(\mathbb{H})$, where $\pi_{1}(\mathbb{H})$ is a $\pi_{0}(\mathbb{G})$-module via $\pi_{0}(T)$, which is given by $u \mapsto \mu_{0} T(u) \mu_{0}^{-1}$.

\section{Semidirect products and split extensions of categorical groups}

If $G$ and $H$ are groups, an action of $G$ on $H$ is a group homomorphism $G \rightarrow$ $A u t(H)$ and, in such case, $H$ is said to be a $G$-group. In the context of crossed modules of groups, K. Norrie [16] introduced the notion of actor of a crossed module, as the analogue of the automorphism group of a group, and then she considered actions of a crossed module on another one. More generally, by considering the categorical group $\mathcal{E} q(\mathbb{H})$ of the equivalences of a categorical group (see $\S 2$, Example 5), we have the following:

Definition 3.1. ([3],[7]) Let $\mathbb{G}$ and $\mathbb{H}$ be categorical groups. An action of $\mathbb{G}$ on $\mathbb{H}$ is a homomorphism of categorical groups $(T, \mu): \mathbb{G} \rightarrow \mathcal{E} q(\mathbb{H})$. When such a $\mathbb{G}$-action is given we shall say that $\mathbb{H}$ is a $\mathbb{G}$-categorical group.

Proposition 3.2. Giving an action of a categorical group $\mathbb{G}$ on another categorical group $\mathbb{H}$ is equivalent to giving a functor

$$
\text { ac: } \mathbb{G} \times \mathbb{H} \longrightarrow \mathbb{H}, \quad(X, A) \mapsto{ }^{X} A,
$$

together with natural isomorphisms

$$
\phi=\phi_{X, Y, A}:(X \otimes Y) A \stackrel{\sim}{\longrightarrow} X\left({ }^{Y} A\right)
$$




$$
\begin{gathered}
\phi_{0}=\phi_{0, A}:{ }^{I} A \stackrel{\sim}{\longrightarrow} A \\
\psi=\psi_{X, A, B}:{ }^{X}(A \otimes B) \stackrel{\sim}{\longrightarrow}{ }^{X} A \otimes{ }^{X} B
\end{gathered}
$$

such that, for any objects $X, Y, Z \in \mathbb{G}$ and $A, B, C \in \mathbb{H}$, the following diagrams are commutative:
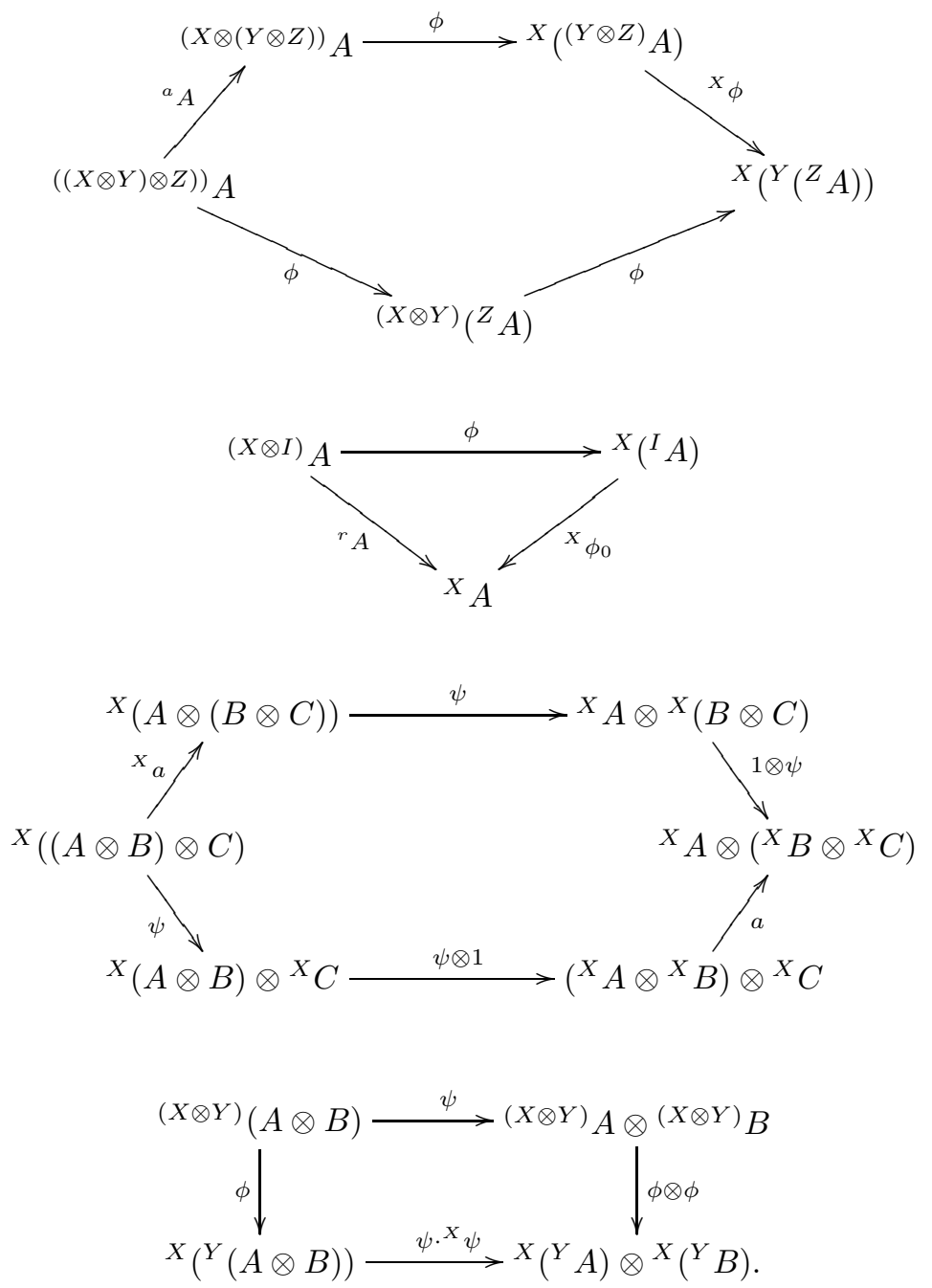

Proof. If $\mathbf{T}=(T, \mu): \mathbb{G} \rightarrow \mathcal{E} q(\mathbb{H})$ is an action of $\mathbb{G}$ on $\mathbb{H}$, we define a functor $a c: \mathbb{G} \times \mathbb{H} \longrightarrow \mathbb{H}$ by letting, for any pair of objects $X \in \mathbb{G}$ and $A \in \mathbb{H}, a c(X, A)=$ ${ }^{X} A=T(X)(A)$, and for any pair of morphisms, $u: X \rightarrow Y$ in $\mathbb{G}$ and $f: A \rightarrow B$ in $\mathbb{H}, a c(u, f)={ }^{u} f$ where ${ }^{u} f$ is the diagonal morphism in the following diagram (which is commutative due to the naturality of $T(u)$ applied to $f$ ) 


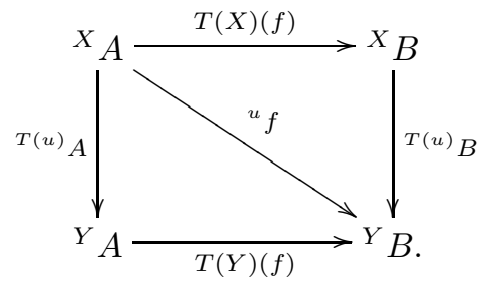

Also, by considering the natural isomorphisms $\phi_{X, Y, A}=\left(\mu_{X, Y}\right)_{A}:{ }^{(X \otimes Y)} A \longrightarrow$ $X\left({ }^{Y} A\right)$, the commutativity of (17) is consequence of (3) and, by considering the isomorphisms $\phi_{0, A}=\left(\mu_{0}\right)_{A}:{ }^{I} A \rightarrow A$, the commutativity of (18) is consequence of (5). Moreover, if for any object $X \in \mathbb{G}$ we consider the equivalence of $\mathbb{H}\left(T(X), \mu_{X}\right)$, we have the natural isomorphisms $\psi_{X, A, B}=\left(\mu_{X}\right)_{A, B}:{ }^{X}(A \otimes B) \rightarrow{ }^{X} A \otimes{ }^{X} B$. These satisfy that (19) is commutative as consequence of (3) and (20) is so because the commutativity of (6) for $\mu_{X, Y}$.

Conversely, suppose given a functor $a c: \mathbb{G} \times \mathbb{H} \rightarrow \mathbb{H}$ together with isomorphisms (14), (15) and (16) satisfying that the diagrams (17), (18), (19) and (20) are commutative. Then, each object $X \in \mathbb{G}$ defines an equivalence of $\mathbb{H}\left(T(X), \mu_{X}\right)$ where $T(X)(A)={ }^{X} A, T(X)(A \stackrel{f}{\rightarrow} B)={ }^{X} A \stackrel{{ }^{X} f}{\longrightarrow} X$ and $\mu_{X}=\left(\mu_{X}\right)_{A, B}:{ }^{X}(A \otimes B) \longrightarrow$ ${ }^{X} A \otimes{ }^{X} B$ is the isomorphism $\psi_{X, A, B}$. In this way, we actually have an equivalence of $\mathbb{H}$ because the property corresponding to (3) is given by (19). Also, each morphism $u: X \rightarrow Y$ in $\mathbb{G}$ defines a natural equivalence $T(u): T(X) \rightarrow T(Y)$ given by $T(u)_{A}={ }^{X} A \stackrel{{ }^{u} A}{\longrightarrow}{ }^{Y} A$ and so we have defined a morphism between the equivalences $\left(T(X), \mu_{X}\right)$ and $\left(T(Y), \mu_{Y}\right)$ because (6) holds as consequence of the naturality of $\psi$. In this way we have a functor $T: \mathbb{G} \rightarrow \mathcal{E} q(\mathbb{H})$ and this determines, together with the natural isomorphisms $\phi_{X, Y, A}:{ }^{(X \otimes Y)} A \longrightarrow{ }^{X}\left({ }^{Y} A\right)$, a homomorphism of categorical groups since (3) and (6) hold as consequence of (17) and (20) respectively.

Note that, for any $X \in \mathbb{G}$, there exists a unique isomorphism

$$
\psi_{0}=\psi_{0, X}:{ }^{X} I \stackrel{\sim}{\longrightarrow} I
$$

determined by the commutativity of the following diagrams:
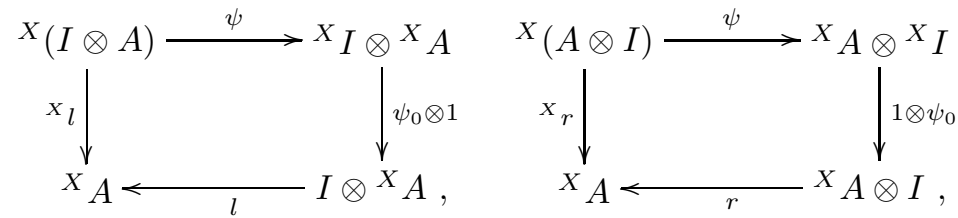

and then the following diagram is also commutative: 


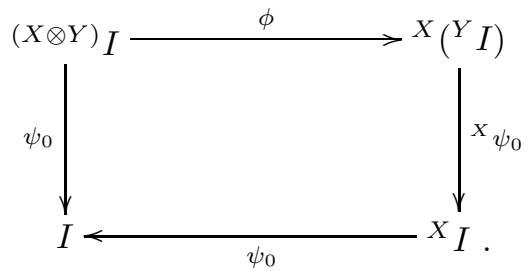

If $G$ and $H$ are groups and we consider the discrete categorical groups they define, $G \rightrightarrows G$ and $H \rightrightarrows H$, then $\mathcal{E} q(H \rightrightarrows H)=(\operatorname{Aut}(H) \rightrightarrows A u t(H))$ and a $(G \rightrightarrows G)$ action on $(H \rightrightarrows H)$ is a $G$-action, in the usual sense, on $H$, that is, a structure of $G$-group on $H$.

Definition 3.3. If $\mathbb{G}$ and $\mathbb{H}$ are categorical groups, two actions of $\mathbb{G}$ on $\mathbb{H}, \mathbf{T}=$ $(T, \mu), \mathbf{T}^{\prime}=\left(T^{\prime}, \mu^{\prime}\right): \mathbb{G} \rightarrow \mathcal{E} q(\mathbb{H})$ are termed equivalent if there exists a morphism $\epsilon: \mathbf{T} \rightarrow \mathbf{T}^{\prime}$.

If $\mathbf{T}$ and $\mathbf{T}^{\prime}$ are actions and, for each $X \in \mathbb{G}$ and $A \in \mathbb{H}$, we denote $T_{X}(A)={ }^{X} A$ and $T_{X}^{\prime}(A)=A^{X}$, the actions

$$
(X, A) \mapsto{ }^{X} A \quad, \quad(X, A) \mapsto A^{X}
$$

are equivalent if, and only if, there exist natural isomorphisms

$$
\epsilon_{X, A}:{ }^{X} A \longrightarrow A^{X}
$$

such that, for any objects $X, Y \in \mathbb{G}$ and $A, B \in \mathbb{H}$, the following diagrams are commutative:

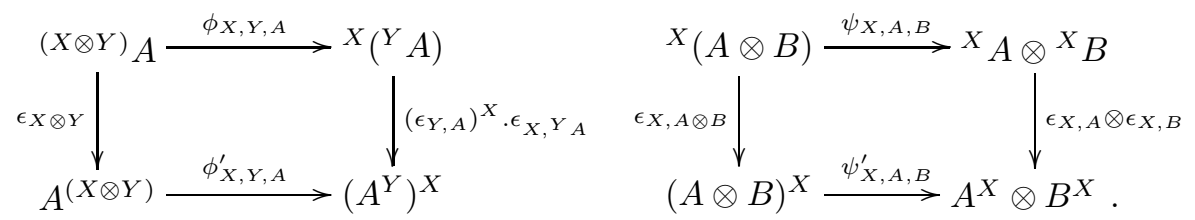

The existence of an equivalence between two actions of $\mathbb{G}$ on $\mathbb{H}$ determines an equivalence relation in the set of all actions of $\mathbb{G}$ on $\mathbb{H}$ and we will denote by $\operatorname{Act}(\mathbb{G}, \mathbb{H})$ the corresponding quotient set.

Now, using the definition of action of a categorical group on another one, we formulate the notion of semidirect product for categorical groups.

Definition 3.4. Let $\mathbb{H}$ be a $\mathbb{G}$-categorical group via an action $(X, A) \mapsto{ }^{X} A$. We define the semidirect product of $\mathbb{H}$ by $\mathbb{G}$ as the categorical group, denoted by $\mathbb{H} \ltimes \mathbb{G}$, whose underlying groupoid is the product $\mathbb{H} \times \mathbb{G}$ with tensor functor given by:

$$
\begin{gathered}
(A, X) \otimes(B, Y)=\left(A \otimes{ }^{X} B, X \otimes Y\right) \\
(u, f) \otimes(v, g)=\left(u \otimes{ }^{f} v, f \otimes g\right) .
\end{gathered}
$$

The unit object is the pair $(I, I)$ and the constraints of associativity and left and right unit are given by: 


$$
\begin{gathered}
a_{(A, X),(B, Y),(C, Z)}=\left(\left(1 \otimes \psi_{X, B,{ }^{Y} C}^{-1}\right)\left(1 \otimes\left(1 \otimes \phi_{X, Y, C}\right)\right) a_{A,{ }^{X}{ }_{B, X}{ }^{X}{ }_{Y} C}, a_{X, Y, Z}\right), \\
l_{(A, X)}=\left(l_{A}\left(1 \otimes \phi_{0, A}\right), l_{X}\right) \quad, \quad r_{(A, X)}=\left(r_{A}\left(1 \otimes \psi_{0, X}\right), r_{X}\right) .
\end{gathered}
$$

The following definition of extension of a categorical group $\mathbb{G}$ by a categorical group $\mathbb{H}$ is due to Breen [3].

Definition 3.5. Let $\mathbb{G}$ and $\mathbb{H}$ be categorical groups. An extension of $\mathbb{G}$ by $\mathbb{H}$ is a sequence of categorical groups and homomorphisms of categorical groups

$$
\mathbb{H} \stackrel{\mathbf{j}}{\longrightarrow} \mathbb{E} \stackrel{\mathbf{p}}{\longrightarrow} \mathbb{G}
$$

such that $p$ is an essentially surjective fibration and $j$ establishes an equivalence between $\mathbb{H}$ and $\mathbb{E}_{I}$, the fiber category of $p$ on the unit object of $\mathbb{G}$ (i.e., $p(A)=I$ if $A \in \mathbb{E}_{I}$, and $p(f)=i d_{I}$ if $f: A \rightarrow B$ is a morphism in $\left.\mathbb{E}_{I}\right)$.

The extension is termed split if there exists a homomorphism of categorical groups $\mathbf{s}: \mathbb{G} \rightarrow \mathbb{E}$ such that $\mathbf{p s}=i d_{\mathbb{G}}$.

Note that if $\mathbb{G}$ and $\mathbb{H}$ are categorical groups, and we consider the product $\mathbb{H} \times \mathbb{G}$, we have a split extension:

$$
\mathbb{H} \stackrel{\mathbf{j}}{\longrightarrow} \mathbb{H} \times \mathbb{G} \underset{\mathrm{s}}{\stackrel{\mathrm{p}}{\rightleftarrows}} \mathbb{G} .
$$

where $j(A)=(A, I), p(A, X)=X$ and $s(X)=(I, X)$, which is called the direct product extension.

Definition 3.6. Suppose $\mathbb{H} \stackrel{\mathbf{j}}{\longrightarrow} \mathbb{E} \stackrel{\mathbf{p}}{\longrightarrow} \mathbb{G}$ and $\mathbb{H} \stackrel{\mathbf{j}^{\prime}}{\longrightarrow} \mathbb{E}^{\prime} \stackrel{\mathbf{p}^{\prime}}{\longrightarrow} \mathbb{G}$ are extensions of $\mathbb{G}$ by $\mathbb{H}$. A morphism between them consists of a pair $(\boldsymbol{\Gamma}, \gamma)$ where $\boldsymbol{\Gamma}: \mathbb{E} \rightarrow \mathbb{E}^{\prime}$ is a homomorphism of categorical groups with $\mathbf{p}^{\prime} \boldsymbol{\Gamma}=\mathbf{p}$, and $\gamma: \boldsymbol{\Gamma} \mathbf{j} \rightarrow \mathbf{j}^{\prime}$ is a morphism such that $p^{\prime}\left(\gamma_{A}\right)=i d_{I}$ for every object $A \in \mathbb{H}$.

Note that if $\mathbb{H} \longrightarrow \mathbf{j} \longrightarrow \mathbb{E} \underset{\mathbf{s}}{\stackrel{\mathbf{p}}{\rightleftarrows}} \mathbb{G}$ is split and $\mathbb{H} \stackrel{\mathbf{j}^{\prime}}{\longrightarrow} \mathbb{E}^{\prime} \stackrel{\mathbf{p}^{\prime}}{\longrightarrow} \mathbb{G}$ is equivalent to it by means of morphism extensions $(\boldsymbol{\Gamma}, \gamma)$, then $\mathbf{p}^{\prime} \boldsymbol{\Gamma} \mathbf{s}=\mathbf{p s}=i d_{\mathbb{G}}$ and so $\Gamma \mathbf{s}$ is an splitting of $\mathbf{p}^{\prime}$.

The existence of a morphism $(\boldsymbol{\Gamma}, \gamma)$ determines (see ([6], Proposition 3.4) an equivalence relation between extensions and we will denote by $\operatorname{Ext}(\mathbb{G}, \mathbb{H})$ the corresponding quotient set and by $\operatorname{Ext}_{\text {split }}(\mathbb{G}, \mathbb{H})$ the subset of the classes of the split extensions of $\mathbb{G}$ by $\mathbb{H}$. These sets are pointed by the class of the direct product extension.

Proposition 3.7. If $\mathbb{G}$ and $\mathbb{H}$ are categorical groups, each action of $\mathbb{G}$ on $\mathbb{H}$ determines, by considering the semidirect product with this action, a split extension (semidirect product extension)

$$
\mathbb{H} \stackrel{\mathbf{j}}{\longrightarrow} \mathbb{H} \ltimes \mathbb{G} \underset{\mathrm{s}}{\stackrel{\mathrm{p}}{\rightleftarrows}} \mathbb{G} .
$$

Moreover, equivalent actions determine equivalent semidirect product extensions. 
Proof. Let $(X, A) \mapsto^{X} A$ be an action of $\mathbb{G}$ on $\mathbb{H}$. Then we consider the semidirect product $\mathbb{H} \ltimes \mathbb{G}$ and the homomorphisms of categorical groups $\mathbf{j}: \mathbb{H} \rightarrow \mathbb{H} \ltimes \mathbb{G}$, $\mathbf{p}: \mathbb{H} \ltimes \mathbb{G} \rightarrow \mathbb{G}$ and $\mathbf{s}: \mathbb{G} \rightarrow \mathbb{H} \ltimes \mathbb{G}$ defined as follows: $\mathbf{j}=(j, \mu)$ is given by $j(A)=(A, I), j(u)=(u, 1)$ and $\mu_{A, B}=\left(1 \otimes \phi_{0, B}^{-1}, l_{I}^{-1}\right) ; \mathbf{p}=(p, \theta)$ is given by $p(A, X)=X, p(u, f)=f$ and $\theta_{(A, X),(B, Y)}=i d_{X \otimes Y} ; \mathbf{s}=(s, \eta)$ is given by $s(X)=$ $(I, X), s(f)=(1, f)$ and $\eta_{X, Y}=\left(\left(1 \otimes \psi_{0, X}^{-1}\right) l_{I}^{-1}, i d_{X \otimes Y}\right)$. With these definitions, it is now straightforward to see that, $\mathbb{H} \stackrel{\mathbf{j}}{\longrightarrow} \mathbb{H} \ltimes \mathbb{G} \underset{\mathbf{s}}{\stackrel{\mathbf{p}}{\rightleftarrows}} \mathbb{G}$ is actually a split extension of $\mathbb{G}$ by $\mathbb{H}$.

Moreover, if $(X, A) \mapsto^{X} A$ and $(X, A) \mapsto A^{X}$ are two equivalent actions of $\mathbb{G}$ on $\mathbb{H}$, there must exist natural isomorphisms $\epsilon_{X, A}:{ }^{X} A \rightarrow A^{X}$ satisfying (21), and then the semidirect product extensions associated to both actions are equivalent since there exists a morphism of extensions $(\boldsymbol{\Gamma}, \gamma)$ where $\boldsymbol{\Gamma}=(\Gamma, \zeta)$ is given by $\Gamma=i d_{\mathbb{H} \times \mathbb{G}}$ and $\zeta_{(A, X),(B, Y)}:\left(A \otimes^{X} B, X \otimes Y\right) \longrightarrow\left(A \otimes B^{X}, X \otimes Y\right)$ is the morphism $\left(i d_{A} \otimes \epsilon_{X, B}, i d_{X \otimes Y}\right)$. Thus it is clear that $\mathbf{p}^{\prime} \boldsymbol{\Gamma}=\mathbf{p}$. On the other hand, $\gamma: \boldsymbol{\Gamma} \mathbf{j} \rightarrow \mathbf{j}^{\prime}$ is the identity since $\mathbf{\Gamma} \mathbf{j}=\mathbf{j}^{\prime}$ as consequence of the equality $\phi_{0, B}^{\prime} \cdot \epsilon_{I, B}=\phi_{0, B}$ which can be deduced from (7).

Now, suppose $\mathbb{H} \stackrel{\mathbf{j}}{\longrightarrow} \mathbb{E} \stackrel{\mathbf{p}}{\longrightarrow} \mathbb{G}$ is an extension of $\mathbb{G}$ by $\mathbb{H}$. Then, for each $A \in \mathbb{E}_{I}$ and each $X \in \mathbb{E}$, once an inverse $X^{*}$ of $X$ has been chosen, we have a morphism (which is the composite of canonical morphisms) $p\left((X \otimes A) \otimes X^{*}\right) \longrightarrow I$. Thus, since $p$ is a fibration, there exists a morphism in $\mathbb{E}, \delta_{A, X}$, with source $(X \otimes A) \otimes X^{*}$ that is mapped by $p$ in the above morphism. The target of this morphism $\delta_{A, X}$ belongs to $\mathbb{E}_{I}$ and, since $j$ is an equivalence, it is of the form $j\left(\xi_{A, X}\right)$ for a unique object $\xi_{A, X} \in \mathbb{H}$. Then we can define a functor $T: \mathbb{E} \rightarrow \mathcal{E} q(\mathbb{H})$ as follows: For any $X \in \mathbb{E}, T(X)=\left(T_{X}, \mu_{X}\right): \mathbb{H} \rightarrow \mathbb{H}$ is defined by $T_{X}(A)=\xi_{j(A), X}, A \in \mathbb{H}$, and if $f: A \rightarrow B$ is a morphism in $\mathbb{H}, T_{X}(f): \xi_{j(A), X} \rightarrow \xi_{j(B), X}$ is the unique morphism determined by the commutativity of the following diagram:

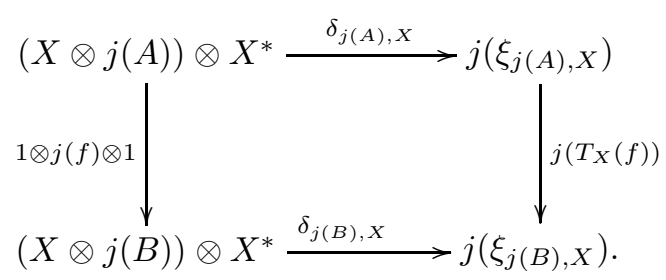

Note that $T_{X}$ is an equivalence of categories with quasi-inverse $T_{X^{*}}$. On the other hand, given $A, B \in \mathbb{H}$, we have to define $\left(\mu_{X}\right)_{A, B}: T_{X}(A \otimes B) \rightarrow T_{X}(A) \otimes T_{X}(B)$, that is, $\left(\mu_{X}\right)_{A, B}: \xi_{j(A \otimes B), X} \rightarrow \xi_{j(A), X} \otimes \xi_{j(B), X}$ and this morphism is the uniquely determined by the commutativity of the following diagram:

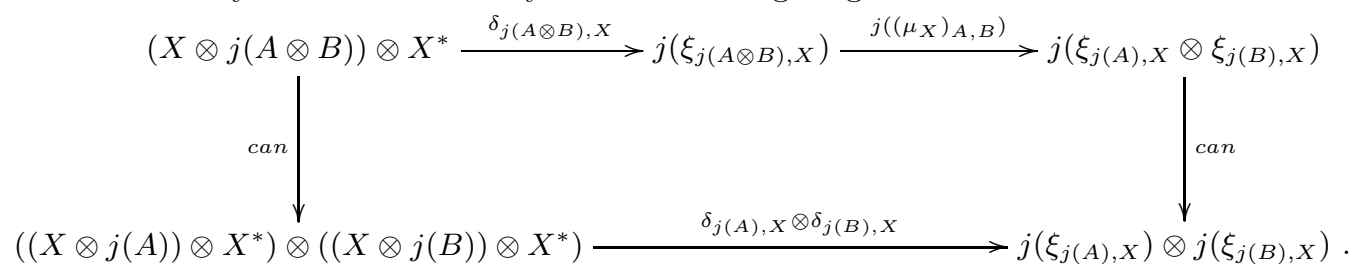


To check that diagrams (3) commute for these morphisms $\left(\mu_{X}\right)_{A, B}$ is routine. Moreover, $\mathbf{T}=(T, \mu): \mathbb{E} \rightarrow \mathcal{E} q(\mathbb{H})$ is a homomorphism of categorical groups where $\mu_{X, Y}: T(X \otimes Y) \rightarrow T(X) \otimes T(Y), X, Y \in \mathbb{E}$, is the morphism in $\mathcal{E} q(\mathbb{H})$ defined by the natural tansformation determined by the morphism in $\mathbb{H}\left(\mu_{X, Y}\right)_{A}$ : $T_{X \otimes Y}(A) \rightarrow T_{X} T_{Y}(A)$, that is, $\left(\mu_{X, Y}\right)_{A}: \xi_{j(A), X \otimes Y} \rightarrow \xi_{j\left(\xi_{j(A), Y}\right), X}$, which is the morphism uniquely determined by the commutativity of the following diagram:

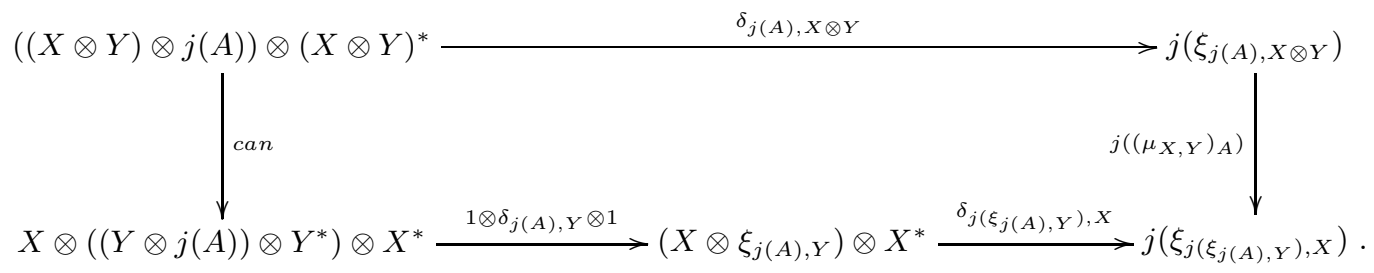

All these facts allow now to show the following:

Proposition 3.8. If $\mathbb{G}$ and $\mathbb{H}$ are categorical groups, each split extension of $\mathbb{G}$ by $\mathbb{H}$,

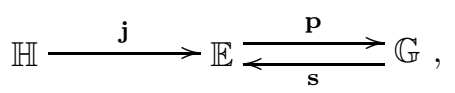

determines an action of $\mathbb{G}$ on $\mathbb{H}$. Moreover, any other equivalent extension of $\mathbb{G}$ by $\mathbb{H}$ determines an equivalent action.

Proof. If we compose the splitting $\mathbf{s}: \mathbb{G} \rightarrow \mathbb{E}$ with the homomorphism $\mathbf{T}: \mathbb{E} \rightarrow$ $\mathcal{E} q(\mathbb{H})$ above described, then we have an action of $\mathbb{G}$ on $\mathbb{H}, \mathbf{F}=\mathbf{T s}: \mathbb{G} \rightarrow \mathcal{E} q(\mathbb{H})$, denoted by ${ }^{X} A$, which is given by ${ }^{X} A=\xi_{j(A), s(X)}$.

If $\mathbb{H} \stackrel{\mathbf{j}^{\prime}}{\longrightarrow} \mathbb{E}^{\prime} \stackrel{\mathbf{p}^{\prime}}{\longrightarrow} \mathbb{G}$ is another extension of $\mathbb{G}$ by $\mathbb{H}$, that is equivalent to the given one by means of morphism extensions $(\boldsymbol{\Gamma}, \gamma)$, then $\boldsymbol{\Gamma} \mathbf{s}$ is an splitting of $\mathbf{p}^{\prime}$. This splitting determines, by composing it with the corresponding homomorphism $\mathbf{T}^{\prime}: \mathbb{E}^{\prime} \rightarrow \mathcal{E} q(\mathbb{H})$, another action of $\mathbb{G}$ on $\mathbb{H}$, denoted by $A^{X}$, and given by $A^{X}=$ $\xi_{j^{\prime}(A), \Gamma s(X)}$.

Thus, the actions ${ }^{X} A$ y $A^{X}$, determined by both split extensions, are equivalent by means of the isomorphisms $\epsilon_{X, A}:{ }^{X} A \rightarrow A^{X}$ determined uniquely by the commutativity of the following diagrams:

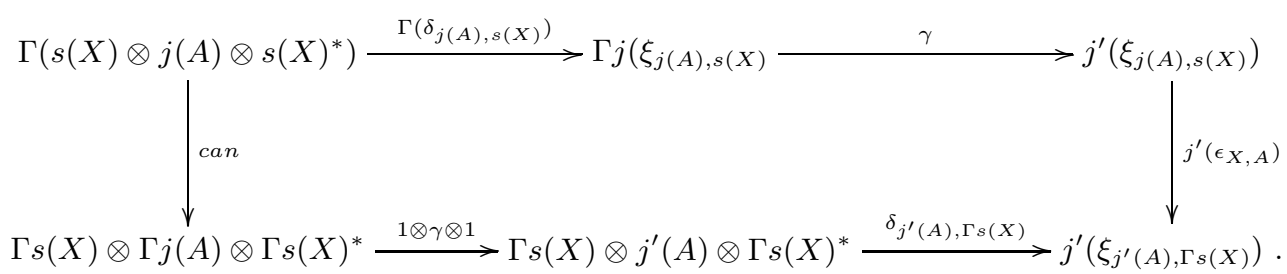

Now it is straightforward to check that these isomorphisms satisfy the commutativity of (21) and therefore we have that the two actions are equivalent. 
Propositions (3.7) and (3.8) assure that we have well-defined maps

$$
\Delta: \operatorname{Act}(\mathbb{G}, \mathbb{H}) \rightarrow \operatorname{Ext}_{\text {split }}(\mathbb{G}, \mathbb{H}) \quad, \quad \Psi: \operatorname{Ext}_{\text {split }}(\mathbb{G}, \mathbb{H}) \rightarrow \operatorname{Act}(\mathbb{G}, \mathbb{H})
$$

and actually we have:

Theorem 3.9. The map

$$
\Delta: \operatorname{Act}(\mathbb{G}, \mathbb{H}) \rightarrow \operatorname{Ext}_{s p l i t}(\mathbb{G}, \mathbb{H})
$$

is a bijection with inverse the map $\Psi$.

Proof. If $\underline{\mathbb{E}}: \mathbb{H} \stackrel{\mathbf{j}}{\longrightarrow} \mathbb{E} \underset{\mathbf{s}}{\stackrel{\mathbf{p}}{\rightleftarrows}} \mathbb{G}$. is a split extension of $\mathbb{G}$ by $\mathbb{H}$, then we have that $\underline{\mathbb{E}}$ and $\Delta \Psi(\underline{\mathbb{E}})=\left(\mathbb{H} \stackrel{\mathbf{j}^{\prime}}{\longrightarrow} \mathbb{H} \ltimes \mathbb{G} \underset{\mathbf{s}^{\prime}}{\stackrel{\mathbf{p}^{\prime}}{\rightleftarrows}} \mathbb{G}\right.$. $)$ are equivalent. To see this, we define a functor $\Gamma: \mathbb{H} \ltimes \mathbb{G} \rightarrow \mathbb{E}$ as follows. Given $(A, X) \in \mathbb{H} \ltimes \mathbb{G}$ we consider the object of $\mathbb{E} j(A) \otimes s(X)$ and the morphism $p(j(A) \otimes s(X)) \stackrel{\mu}{\longrightarrow} I \otimes X \stackrel{l}{\longrightarrow}$ $X$ where $\mathbf{p}=(p, \mu)$; then, since $p$ is a fibration, there exists a morphism in $\mathbb{E}$ with source $j(A) \otimes s(X)$ which is carried by $p$ in the morphism $l \mu$; if $\lambda_{A, X}$ is such a morphism, we define $\Gamma(A, X)$ as the target of $\lambda_{A, X}$, and this morphism satisfies that $p \Gamma(A, X)=X$. Given $(u, f):(A, X) \rightarrow(Y, B)$ we define $\Gamma(u, f): \Gamma(A, X) \rightarrow \Gamma(B, Y)$ as the morphism $\lambda_{B, Y}(j(u) \otimes s(f)) \lambda_{A, X}^{-1}$ and, in this way, it is clear that $\Gamma$ is actually a functor. Moreover, $\Gamma=(\Gamma, \theta)$ is a homomorphism of categorical groups where $\theta_{(A, X),(B, Y)}: \Gamma((A, X) \otimes(B, Y)) \rightarrow \Gamma(A, X) \otimes \Gamma(B, Y)$ is defined as follows: $(A, X) \otimes(B, Y)=\left(A \otimes{ }^{X} B, X \otimes Y\right)=\left(A \otimes \xi_{j(B), s(X)}, X \otimes Y\right)$ and $\Gamma((A, X) \otimes(B, Y))$ is then the target of the following morphism

$$
\lambda_{A \otimes \xi_{j(B), s(X)}, X \otimes Y}: j\left(A \otimes \xi_{j(B), s(X)} \otimes s(X \otimes Y) \rightarrow \Gamma((A, X) \otimes(B, Y)) ;\right.
$$

also, we have the morphism $\lambda_{A, X} \otimes \lambda_{B, Y}: j(A) \otimes s(X) \otimes j(B) \otimes s(Y) \rightarrow \Gamma(A, X) \otimes$ $\Gamma(B, Y)$ and if $\zeta$ is the following isomorphism, given by composition of canonical isomorphisms,

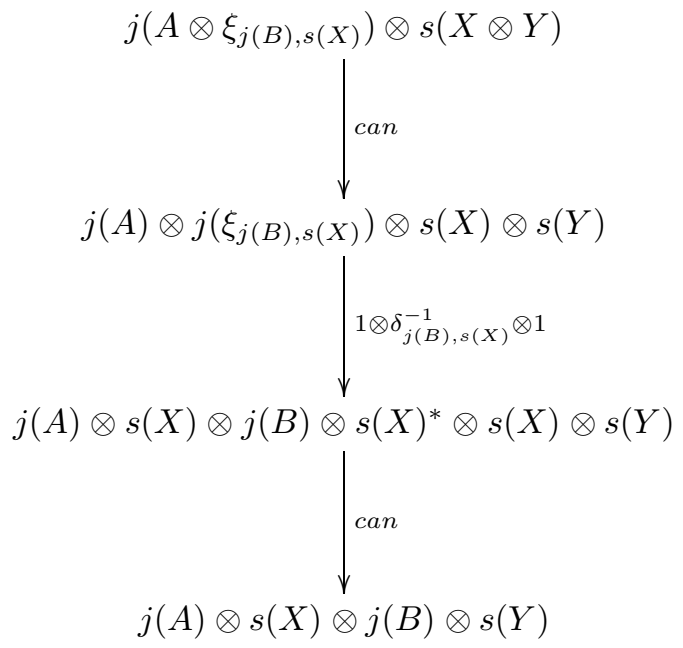


we define $\theta_{(A, X),(B, Y)}=\left(\lambda_{A, X} \otimes \lambda_{B, Y}\right) \zeta \lambda_{A \otimes \xi_{j(B), s(X)}, X \otimes Y}^{-1}$. Thus $\mathbf{p} \boldsymbol{\Gamma}=\mathbf{p}^{\prime}$. Moreover, there exists a morphism $\gamma: \boldsymbol{\Gamma j}^{\prime} \rightarrow \mathbf{j}$ that is given, for any object $A \in \mathbb{H}$, as the following composition:

$$
\gamma_{A}: \Gamma(A, I) \stackrel{\gamma_{A, I}^{-1}}{\longrightarrow} j(A) \otimes s(I) \stackrel{1 \otimes \mu_{0}}{\longrightarrow} j(A) \otimes I \stackrel{r}{\longrightarrow} j(A) .
$$

Note that $\gamma$ is natural since, for any morphism $u: A \rightarrow A^{\prime}$, we have that

$$
\begin{aligned}
\gamma_{A^{\prime}} \Gamma j^{\prime}(u) & =r\left(1 \otimes \mu_{0}\right) \lambda_{A^{\prime}, I}^{-1} \Gamma j^{\prime}(u) \\
& =r\left(1 \otimes \mu_{0}\right) \lambda_{A^{\prime}, I}^{-1} \lambda_{A^{\prime}, I}(j(u) \otimes s(1)) \lambda_{A, I}^{-1} \\
& =r\left(1 \otimes \mu_{0}\right)(j(u) \otimes 1) \lambda_{A, I}^{-1} \\
& =r(j(u) \otimes 1)\left(1 \otimes \mu_{0}\right) \lambda_{A, I}^{-1} \\
& =j(u) r\left(1 \otimes \mu_{0}\right) \lambda_{A, I}^{-1} \\
& =j(u) \gamma_{A}
\end{aligned}
$$

and to check the commutativity of (6) is straightforward.

Conversely, given an action $T: \mathbb{G} \rightarrow \mathcal{E} q(\mathbb{H})$, denoted by ${ }^{X} A$, we have

$$
\Psi \Delta(T)=\Psi(\mathbb{H} \stackrel{\mathbf{j}}{\longrightarrow} \mathbb{H} \ltimes \mathbb{G} \underset{\mathrm{s}}{\stackrel{\mathrm{p}}{\rightleftarrows}} \mathbb{G} .)=(\mathbb{G} \rightarrow \mathcal{E} q(\mathbb{H}))
$$

and we denote this action by $A^{X}$ that is given in the target of the following morphism:

$$
\delta_{j(A), s(X)}:(I, X) \otimes(A, I) \otimes(I, X)^{*} \longrightarrow\left(A^{X}, I\right) .
$$

Now, since $(I, X) \otimes(A, I) \otimes(I, X)^{*}=\left(I \otimes \otimes^{X} A \otimes{ }^{X} \otimes I I, X \otimes I \otimes X^{*}\right)$, this new action is equivalent to the first one by means of the isomorphisms $\epsilon_{X, A}:{ }^{X} A \rightarrow A^{X}$ which are defined as the following composition of canonical isomorphisms:

$$
\begin{gathered}
\left({ }^{X} A, I\right) \rightarrow\left(I \otimes{ }^{X} A \otimes I, I\right) \rightarrow\left(I \otimes{ }^{X} A \otimes{ }^{X} I, X \otimes X^{*}\right) \rightarrow \\
\left(I \otimes{ }^{X} A \otimes{ }^{X \otimes I} I, X \otimes I \otimes X^{*}\right) \rightarrow\left(A^{X}, I\right) .
\end{gathered}
$$

Finally it is straightforward to check that diagrams (21) are commutative and the proof is finished.

\section{Obstruction theory}

Let $\mathbb{H}$ be a categorical group and $\mathcal{E} q(\mathbb{H})$ the categorical group of the equivalences of $\mathbb{H}$ (see $\S 2$, Example 5 ). We consider the group $\pi_{0}(\mathcal{E} q(\mathbb{H})$ ) whose elements are the isomorphism classes of equivalences of $\mathbb{H}$ with product induced by the composition of equivalences, that is, $[\mathbf{T}]\left[\mathbf{T}^{\prime}\right]=\left[\mathbf{T} \mathbf{T}^{\prime}\right]$, for any equivalences $\mathbf{T}=(T, \mu), \mathbf{T}^{\prime}=$ $\left(T^{\prime}, \mu^{\prime}\right): \mathbb{H} \rightarrow \mathbb{H}$. Also, we will consider the abelian group $\pi_{1}(\mathcal{E} q(\mathbb{H}))$ whose elements are the morphisms $u: i d_{\mathbb{H}} \rightarrow i d_{\mathbb{H}}$. Thus, an element $u \in \pi_{1}(\mathcal{E} q(\mathbb{H}))$ consists of a family of automorphisms $u_{A}: A \rightarrow A, A \in \mathbb{H}$, such that $f u_{A}=u_{B} f$ for any morphism $f: A \rightarrow B$ in $\mathbb{H}$, and such that $u_{A \otimes B}=u_{A} \otimes u_{B}$ for any objects $A, B \in \mathbb{H}$.

Let us note that, for any $u \in \pi_{1}(\mathcal{E} q(\mathbb{H}))$ and any morphism $\epsilon: \mathbf{T} \rightarrow \mathbf{T}^{\prime}$, where $\mathbf{T}$ 
and $\mathbf{T}^{\prime}$ are equivalences of $\mathbb{H}$, we have the equality

$$
u T^{\prime} \cdot \epsilon=\epsilon \cdot u T
$$

and therefore, as consequence of (10), we have the following:

Lemma 4.1. Let $\mathbf{T}, \mathbf{T}^{\prime}: \mathbb{H} \rightarrow \mathbb{H}$ be equivalences of the categorical group $\mathbb{H}$. Then, for any two isomorphisms $\theta, \epsilon: \mathbf{T} \rightarrow \mathbf{T}^{\prime}$, there exists a unique $u \in \pi_{1}(\mathcal{E} q(\mathbb{H}))$ such that

$$
\epsilon=u T^{\prime} \cdot \theta=\theta \cdot u T
$$

Let us recall that, according to $(11), \pi_{1}(\mathcal{E} q(\mathbb{H}))$ is a $\pi_{0}(\mathcal{E} q(\mathbb{H}))$-module, that is, there exists a group homomorphism $\rho: \pi_{0}(\mathcal{E} q(\mathbb{H})) \rightarrow \operatorname{Aut}\left(\pi_{1}(\mathcal{E} q(\mathbb{H}))\right)$ given by $\rho_{[T]}(u)={ }^{T} u$, where ${ }^{T} u$ is the unique element of $\pi_{1}(\mathcal{E} q(\mathbb{H}))$ such that

$$
{ }^{T} u T=T u .
$$

Now, keeping the Baer notion of "kollektivcharacter" in mind, we shall define a collective character of a categorical group $\mathbb{G}$ in a categorical group $\mathbb{H}$ as a pair $(\varphi, \psi)$ where $\varphi: \pi_{0}(\mathbb{G}) \rightarrow \pi_{0}(\mathcal{E} q(\mathbb{H}))$ is a group homomorphism and $\psi: \pi_{1}(\mathbb{G}) \rightarrow$ $\pi_{1}(\mathcal{E} q(\mathbb{H}))$ is a homomorphism of $\pi_{0}(\mathbb{G})$-modules, where $\pi_{1}(\mathcal{E} q(\mathbb{H}))$ is so via $\varphi$, that is, through the induced homomorphism

$$
\operatorname{Hom}_{G p}\left(\pi_{0}(\mathbb{G}), \pi_{0}(\mathcal{E} q(\mathbb{H}))\right) \stackrel{\rho_{*}}{\longrightarrow} \operatorname{Hom}_{G p}\left(\pi_{0}(\mathbb{G}), \operatorname{Aut}\left(\pi_{1}(\mathcal{E} q(\mathbb{H}))\right)\right), \quad \varphi \mapsto \rho_{*}(\varphi)=\rho \varphi .
$$

Thus, given $x \in \pi_{0}(\mathbb{G})$ and $u \in \pi_{1}(\mathcal{E} q(\mathbb{H}))$, we have that, for any $T \in \varphi(x)$,

$$
{ }^{x} T={ }^{T} u \text {. }
$$

If $(\varphi, \psi)$ is a collective character of $\mathbb{G}$ in $\mathbb{H}$ we will denote by $H_{\varphi}^{n}\left(\pi_{0}(\mathbb{G}), \pi_{1}(\mathcal{E} q(\mathbb{H}))\right)$, $n \geqslant 0$, the $n$-th cohomology group of $\pi_{0}(\mathbb{G})$ with coefficients in the $\pi_{0}(\mathbb{G})$-module (via $\varphi) \pi_{1}(\mathcal{E} q(\mathbb{H}))$. Next we will show that $(\varphi, \psi)$ has canonically associated with it a cohomology class $k(\varphi, \psi) \in H_{\varphi}^{3}\left(\pi_{0}(\mathbb{G}), \pi_{1}(\mathcal{E} q(\mathbb{H}))\right)$ whose construction is analogous to a classic construction by Teichmller [20] for a similar situation with linear algebras, and to that by Eilenberg-Mac Lane [11] for the study of obstructions to group extensions with non-abelian kernels.

Any collective character has associated a functor $T: \mathbb{G} \rightarrow \mathcal{E} q(\mathbb{H})$ which is defined as follows. If $X \in \mathbb{G}$ let us consider $x=[X] \in \pi_{0}(\mathbb{G})$ and $\varphi(x) \in \pi_{0}(\mathcal{E} q(\mathbb{H}))$ and let us choose an equivalence $T_{x}: \mathbb{H} \rightarrow \mathbb{H}$. In particular, if $1=[I]$, select $T_{1}=i d_{\mathbb{H}}$. Then we define $T(X)=T_{x}$. To define $T$ on morphisms, let us choose, in each class $x \in \pi_{0}(\mathbb{G})$, an object $X_{x} \in x$ with $X_{1}=I$ and for any $X \in x$ we select a morphism $i_{X}: X \rightarrow X_{x}$ in $\mathbb{G}$ such that $i_{X_{x}}=i d_{X_{x}}, i_{I \otimes X_{x}}=l_{X_{x}}$ and $i_{X_{x} \otimes I}=r_{X_{x}}$. Then, given any morphism $f: X \rightarrow Y$ in $\mathbb{G}$, if $x=[X]=[Y]$, we consider the automorphism of $X_{x}$

$$
X_{x} \stackrel{i_{X}^{-1}}{\longrightarrow} X \stackrel{f}{\longrightarrow} Y \stackrel{i_{Y}}{\longrightarrow} X_{x}
$$

and using the isomorphism $(8) \gamma_{X_{x}}: \pi_{1}(\mathbb{G}) \rightarrow A u t_{\mathbb{G}}\left(X_{x}\right)$ we define $T(f)=$ $\left(\psi \gamma_{X_{x}}^{-1}\left(i_{Y} f i_{X}^{-1}\right)\right) T_{x}: T_{x} \rightarrow T_{x}$ which is actually a morphism in $\mathcal{E} q(\mathbb{H})$ from $T_{x}$ to $T_{x}$. 
In this way $T$ is a functor since:

$$
\begin{gathered}
T\left(i d_{X}\right)=\left(\psi \gamma_{X_{x}}^{-1}\left(i_{X} i d_{X} i_{X}^{-1}\right)\right) Y_{x}=\left(\psi \gamma_{X_{x}}^{-1}\left(i d_{X_{x}}\right) T_{x}=\left(\psi\left(i d_{I}\right)\right) T_{x}=\right. \\
i d_{i d_{\mathbb{H}}} T_{x}=i d_{T_{x}}=i d_{T(X)},
\end{gathered}
$$

and given morphisms in $\mathbb{G}, X \stackrel{f}{\rightarrow} Y \stackrel{g}{\rightarrow} Z$, we have that

$$
\begin{aligned}
T(g f) & =\left(\psi \gamma_{X_{x}}^{-1}\left(i_{Z} g f i_{X}^{-1}\right)\right) T_{x} \\
& =\left(\psi \gamma_{X_{x}}^{-1}\left(i_{Z} g i_{Y}^{-1} i_{Y} f i_{X}^{-1}\right) T_{x}\right. \\
& =\left(\psi \gamma_{X_{x}}^{-1}\left(i_{Z} g i_{Y}^{-1}\right) \psi \gamma_{X_{x}}^{-1}\left(i_{Y} f i_{X}^{-1}\right)\right) T_{x} \\
& =\left(\psi \gamma_{X_{x}}^{-1}\left(i_{Z} g i_{Y}^{-1}\right)\right) T_{x} \cdot\left(\psi \gamma_{X_{x}}^{-1}\left(i_{Y} f i_{X}^{-1}\right)\right) T_{x} \\
& =T(g) T(f)
\end{aligned}
$$

Let us note that, if $f: X \rightarrow Y$ is a morphism in $\mathbb{G}$ and $Z \in \mathbb{G}$, by using (9), we have that $\alpha=\gamma_{X_{x z}}^{-1}\left(i_{Y \otimes Z}\left(f \otimes i d_{Z}\right) i_{X \otimes Z}^{-1}\right)=\gamma_{X_{x z}}^{-1}\left(\left(i_{Y} f i_{X}^{-1}\right) \otimes i d_{X_{z}}\right)=\gamma_{X_{x}}^{-1}\left(i_{Y} f i_{X}^{-1}\right)$ and thus

$$
T\left(f \otimes i d_{Z}\right)=\psi(\alpha) T_{x z}, \quad T(f)=\psi(\alpha) T_{x} .
$$

Likewise, if $X \in \mathbb{G}$ and $f: Y \rightarrow Z$ is a morphism in $\mathbb{G}$, by using (2), we have that $\beta=\gamma_{X_{x y}}^{-1}\left(i_{X \otimes Z}\left(i d_{X} \otimes f\right) i_{X \otimes Y}^{-1}\right)=\gamma_{X_{x y}}^{-1}\left(i d_{X_{x}} \otimes\left(i_{Z} f i_{Y}^{-1}\right)\right)={ }^{x} \gamma_{X_{y}}^{-1}\left(i_{Z} f i_{Y}^{-1}\right)$ and so, since $\psi$ is a homomorphism of $\pi_{0}(\mathbb{G})$-modules, $\psi(\beta)=\varphi(x) \psi\left(\gamma_{X_{y}}^{-1}\left(i_{Z} f i_{Y}^{-1}\right)\right)=$ ${ }^{\varphi(x)} u$. Thus, if $T(f)=u T_{y}$, we have that

$$
T\left(i d_{X} \otimes f\right)=\psi(\beta) T_{x y}={ }^{(x)} u T_{x y} .
$$

Suppose now $X, Y \in \mathbb{G}$. If $x=[X]$ and $y=[Y]$ then $[X \otimes Y]=[X][Y]=x y$ and so, since $\varphi$ is a group homomorphism, $\varphi(x y)=\varphi(x) \varphi(y)$. Taking into account that in $\mathcal{E} q(\mathbb{H})$ the tensor on objects is given by the composition of equivalences, we have that the equivalences $T_{x y}$ and $T_{x} T_{y}$ belong to the same class in $\pi_{0}(\mathcal{E} q(\mathbb{H}))$. Then we can select isomorphisms

$$
\mu_{x, y}: T_{x y} \rightarrow T_{x} T_{y}
$$

with $\mu_{1, x}=\mu_{x, 1}=i d_{T_{x}}, x \in \pi_{0}(\mathbb{G})$.

For any three objects $X, Y, Z \in \mathbb{G}$, if $x=[X], y=[Y]$ and $z=[Z]$, the following diagram need not be commutative

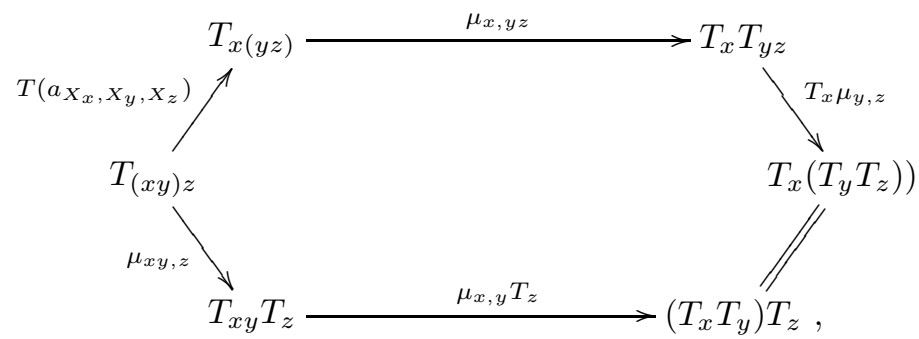

but, by Lemma 4.1, there exists a unique element $k_{x, y, z}^{\varphi, \psi} \in \pi_{1}(\mathcal{E} q(\mathbb{H}))$ such that: 


$$
\mu_{x, y} T_{z} \cdot \mu_{x y, z}=T_{x} \mu_{y, z} \cdot \mu_{x, y z} \cdot T\left(a_{X_{x}, X_{y}, X_{z}}\right) \cdot k_{x, y, z}^{\varphi, \psi} T_{(x y) z}
$$

According to the choices we have made, we have clearly that $k_{x, y, z}^{\varphi, \psi}$ determines a normalized 3-dimensional cochain of $\pi_{0}(\mathbb{G})$ with coefficients in $\pi_{1}(\mathcal{E} q(\mathbb{H}))$. Even more:

Proposition 4.2. The cochain $k^{\varphi, \psi}: \pi_{0}(\mathbb{G})^{3} \rightarrow \pi_{1}(\mathcal{E} q(\mathbb{H}))$ is a 3-cocycle of $\pi_{0}(\mathbb{G})$ with coefficients in the $\pi_{0}(\mathbb{G})$-module $\pi_{1}(\mathcal{E} q(\mathbb{H}))$. Moreover:

i) If the choice of $\left\{X_{x}, i_{X}\right\}$ (for the definition of $T$ on morphisms) is modified, then $k^{\varphi, \psi}$ changes to a cohomologous 3-cocycle.

ii) If the choice of $\mu$ in (27) is modified, then $k^{\varphi, \psi}$ changes to a cohomologous 3cocycle and, by suitably changing $\mu, k^{\varphi, \psi}$ may be changed to any cohomologous 3-cocycle.

iii) If the choice of the equivalences $T_{x}$ (for the definition of $T$ on objects) is modified, then a suitable new selection of $\mu$ leaves the 3 -cocycle $k^{\varphi, \psi}$ unaltered.

Proof. To prove that $k^{\varphi, \psi}$ is a 3 -cocycle, let $(x, y, z, w) \in \pi_{0}(\mathbb{G})^{4}$. Then we compute the isomorphism

$$
J: T_{x y z w} \stackrel{\mu_{(x y) z, w}}{\longrightarrow} T_{x y z} T_{w} \stackrel{\mu_{x y, z} T_{w}}{\longrightarrow} T_{x y} T_{z} T_{w} \stackrel{\mu_{x, y} T_{z} T_{w}}{\longrightarrow} T_{x} T_{y} T_{z} T_{w}
$$

in two ways. On the one hand, we have:

$$
\begin{aligned}
J \stackrel{(29)}{=} & \mu_{x, y} T_{z} T_{w} \cdot T_{x y} \mu_{z, w} \cdot \mu_{x y, z w} \cdot T\left(a_{X_{x}, X_{y}, X_{z}}\right) \cdot k_{x y, z, w}^{\varphi, \psi} T_{((x y) z) w} \\
\stackrel{(\text { nat })}{=} & T_{x} T_{y} \mu_{z, w} \cdot \mu_{x, y} T_{z w} \cdot \mu_{x y, z w} \cdot T\left(a_{X_{x y}, X_{z} X_{w}}\right) \cdot k_{x y, z, w}^{\varphi, \psi} T_{((x y) z) w} \\
\stackrel{(29)}{=} & T_{x} T_{y} \mu_{z, w} \cdot T_{x} \mu_{y, z w} \cdot \mu_{x, y(z w)} \cdot T\left(a_{X_{x}, X_{y}, X_{z w}}\right) \cdot k_{x, y, z w}^{\varphi, \psi} T_{(x y)(z w)} \\
& T\left(a_{\left.X_{x y}, X_{z} X_{w}\right) \cdot k_{x y, z, w}^{\varphi, \psi} T_{((x y) z) w}}\right. \\
& \stackrel{(23)}{=} \quad T_{x} T_{y} \mu_{z, w} \cdot T_{x} \mu_{y, z w} \cdot \mu_{x, y(z w)} \cdot T\left(a_{X_{x}, X_{y}, X_{z w}}\right) \cdot T\left(a_{X_{x y}, X_{z} X_{w}}\right) . \\
& k_{x, y, z w}^{\varphi, \psi} T_{((x y) z) w} \cdot k_{x y, z, w}^{\varphi, \psi} T_{((x y) z) w} \\
= & T_{x} T_{y} \mu_{z, w} \cdot T_{x} \mu_{y, z w} \cdot \mu_{x, y(z w)} \cdot T\left(a_{X_{x}, X_{y}, X_{z w}}\right) \cdot T\left(a_{X_{x y}, X_{z} X_{w}}\right) . \\
& \left(k_{x, y, z w}^{\varphi, \psi} \cdot k_{x y, z, w}^{\varphi, \psi}\right) T_{((x y) z) w}
\end{aligned}
$$

and on the other hand: 


$$
\begin{aligned}
& J \quad=\quad\left(\left(\mu_{x, y} T_{z} \cdot \mu_{x y, z}\right) T_{w}\right) \mu_{(x y) z, w} \\
& \stackrel{(29)}{=} \quad\left(\left(T_{x} \mu_{y, z} \cdot \mu_{x, y z} \cdot T\left(a_{X_{x}, X_{y}, X_{z}}\right) \cdot k_{x, y, z}^{\varphi, \psi} T_{(x y) z}\right) T_{w}\right) \cdot \mu_{(x y) z, w} \\
& =\quad T_{x} \mu_{y, z} T_{w} \cdot \mu_{x, y z} T_{w} \cdot T\left(a_{X_{x}, X_{y}, X_{z}}\right) T_{w} \cdot k_{x, y, z}^{\varphi, \psi} T_{(x y) z} T_{w} \cdot \mu_{(x y) z, w} \\
& \stackrel{(23)}{=} \quad T_{x} \mu_{y, z} T_{w} \cdot \mu_{x, y z} T_{w} \cdot T\left(a_{X_{x}, X_{y}, X_{z}}\right) T_{w} \cdot \mu_{(x y) z, w} \cdot k_{x, y, z}^{\varphi, \psi} T_{((x y) z) w} \\
& \stackrel{(23,25)}{=} T_{x} \mu_{y, z} T_{w} \cdot \mu_{x, y z} T_{w} \cdot \mu_{(x y) z, w} \cdot T\left(a_{X_{x}, X_{y}, X_{z}} \otimes i d_{X_{w}}\right) \cdot k_{x, y, z}^{\varphi, \psi} T_{((x y) z) w} \\
& \stackrel{(29)}{=} T_{x} \mu_{y, z} T_{w} \cdot T_{x} \mu_{y z, w} \cdot \mu_{x,(y z) w} \cdot T\left(a_{X_{x}, X_{y z}, X_{w}}\right) \cdot k_{x, y z, w}^{\varphi, \psi} T_{(x(y z)) w} \cdot \\
& T\left(a_{X_{x}, X_{y}, X_{z}} \otimes i d_{X_{w}}\right) \cdot k_{x, y, z}^{\varphi, \psi} T_{((x y) z) w} \\
& \stackrel{(23)}{=} \quad T_{x} \mu_{y, z} T_{w} \cdot T_{x} \mu_{y z, w} \cdot \mu_{x,(y z) w} \cdot T\left(a_{X_{x}, X_{y z}, X_{w}}\right) \cdot T\left(a_{X_{x}, X_{y}, X_{z}} \otimes i d_{X_{w}}\right) \text {. } \\
& k_{x, y z, w}^{\varphi, \psi} T_{((x y) z) w} \cdot k_{x, y, z}^{\varphi, \psi} T_{((x y) z) w} \\
& =T_{x}\left(\mu_{y, z} T_{w} \cdot \mu_{y z, w}\right) \cdot \mu_{x,(y z) w} \cdot T\left(a_{X_{x}, X_{y z}, X_{w}}\right) \cdot T\left(a_{X_{x}, X_{y}, X_{z}} \otimes i d_{X_{w}}\right) \text {. } \\
& k_{x, y z, w}^{\varphi, \psi} T_{((x y) z) w} \cdot k_{x, y, z}^{\varphi, \psi} T_{((x y) z) w} \\
& \stackrel{(29)}{=} \quad T_{x} T_{y} \mu_{z, w} \cdot T_{x} \mu_{y, z w} \cdot T_{x} T\left(a_{X_{y}, X_{z}, X_{w}}\right) \cdot T_{x} k_{y, z, w}^{\varphi, \psi} T_{(y z) w} \cdot \mu_{x,(y z) w} \cdot \\
& T\left(a_{X_{x}, X_{y z}, X_{w}}\right) \cdot T\left(a_{X_{x}, X_{y}, X_{z}} \otimes i d_{X_{w}}\right) \cdot k_{x, y z, w}^{\varphi, \psi} T_{((x y) z) w} \cdot k_{x, y, z}^{\varphi, \psi} T_{((x y) z) w} \\
& \stackrel{(24)}{=} \quad T_{x} T_{y} \mu_{z, w} \cdot T_{x} \mu_{y, z w} \cdot T_{x} T\left(a_{X_{y}, X_{z}, X_{w}}\right) \cdot{ }^{x} k_{y, z, w}^{\varphi, \psi} T_{x} T_{(y z) w} \cdot \mu_{x,(y z) w} \text {. } \\
& T\left(a_{X_{x}, X_{y z}, X_{w}}\right) \cdot T\left(a_{X_{x}, X_{y}, X_{z}} \otimes i d_{X_{w}}\right) \cdot k_{x, y z, w}^{\varphi, \psi} T_{((x y) z) w} \cdot k_{x, y, z}^{\varphi, \psi} T_{((x y) z) w} \\
& \stackrel{(23)}{=} T_{x} T_{y} \mu_{z, w} \cdot T_{x} \mu_{y, z w} \cdot T_{x} T\left(a_{X_{y}, X_{z}, X_{w}}\right) \cdot \mu_{x,(y z) w} \cdot{ }^{x} k_{y, z, w}^{\varphi, \psi} T_{x((y z) w)} . \\
& T\left(a_{X_{x}, X_{y z}, X_{w}}\right) \cdot T\left(a_{X_{x}, X_{y}, X_{z}} \otimes i d_{X_{w}}\right) \cdot\left(k_{x, y z, w}^{\varphi, \psi} \cdot k_{x, y, z}^{\varphi, \psi}\right) T_{((x y) z) w} \\
& \stackrel{(24)}{=} T_{x} T_{y} \mu_{z, w} \cdot T_{x} \mu_{y, z w} \cdot{ }^{x} T\left(a_{X_{y}, X_{z}, X_{w}}\right) T_{x} \cdot \mu_{x,(y z) w} \cdot{ }^{x} k_{y, z, w}^{\varphi, \psi} T_{x((y z) w)} \cdot \\
& T\left(a_{X_{x}, X_{y z}, X_{w}}\right) \cdot T\left(a_{X_{x}, X_{y}, X_{z}} \otimes i d_{X_{w}}\right) \cdot\left(k_{x, y z, w}^{\varphi, \psi} \cdot k_{x, y, z}^{\varphi, \psi}\right) T_{((x y) z) w} \\
& \stackrel{(23,26)}{=} T_{x} T_{y} \mu_{z, w} \cdot T_{x} \mu_{y, z w} \cdot \mu_{x,(y z) w} \cdot T\left(i d_{X_{x}} \otimes a_{X_{y}, X_{z}, X_{w}}\right) \cdot{ }^{x} k_{y, z, w}^{\varphi, \psi} T_{x((y z) w)} . \\
& T\left(a_{X_{x}, X_{y z}, X_{w}}\right) \cdot T\left(a_{X_{x}, X_{y}, X_{z}} \otimes i d_{X_{w}}\right) \cdot\left(k_{x, y z, w}^{\varphi, \psi} \cdot k_{x, y, z}^{\varphi, \psi}\right) T_{((x y) z) w} \\
& \stackrel{(23)}{=} T_{x} T_{y} \mu_{z, w} \cdot T_{x} \mu_{y, z w} \cdot \mu_{x,(y z) w} \cdot T\left(i d_{X_{x}} \otimes a_{X_{y}, X_{z}, X_{w}}\right) \cdot T\left(a_{X_{x}, X_{y z}, X_{w}}\right) \text {. } \\
& T\left(a_{X_{x}, X_{y}, X_{z}} \otimes i d_{X_{w}}\right) \cdot{ }^{x} k_{y, z, w}^{\varphi, \psi} T_{((x y) z) w)} \cdot\left(k_{x, y z, w}^{\varphi, \psi} \cdot k_{x, y, z}^{\varphi, \psi}\right) T_{((x y) z) w} \\
& =T_{x} T_{y} \mu_{z, w} \cdot T_{x} \mu_{y, z w} \cdot \mu_{x,(y z) w} \text {. } \\
& T\left(\left(i d_{X_{x}} \otimes a_{X_{y}, X_{z}, X_{w}}\right) \cdot a_{X_{x}, X_{y z}, X_{w}} \cdot\left(a_{X_{x}, X_{y}, X_{z}} \otimes i d_{X_{w}}\right)\right) \cdot \\
& \left({ }^{x} k_{y, z, w}^{\varphi, \psi} \cdot k_{x, y z, w}^{\varphi, \psi} \cdot k_{x, y, z}^{\varphi, \psi}\right) T_{((x y) z) w} \\
& \stackrel{(1)}{=} \quad T_{x} T_{y} \mu_{z, w} \cdot T_{x} \mu_{y, z w} \cdot \mu_{x,(y z) w} \cdot T\left(a_{X_{x}, X_{y}, X_{z w}} \cdot a_{X_{x y}, X_{z}, X_{w}}\right) \cdot \\
& \left({ }^{x} k_{y, z, w}^{\varphi, \psi} \cdot k_{x, y z, w}^{\varphi, \psi} \cdot k_{x, y, z}^{\varphi, \psi}\right) T_{((x y) z) w} .
\end{aligned}
$$

Hence, comparison and Lemma 4.1 give

$$
{ }^{x} k_{y, z, w}^{\varphi, \psi} \cdot k_{x, y z, w}^{\varphi, \psi} \cdot k_{x, y, z}^{\varphi, \psi}=k_{x, y, z w}^{\varphi, \psi} \cdot k_{x y, z, w}^{\varphi, \psi}
$$

that is, $k^{\varphi, \psi}$ is a 3 -cocycle.

i) If the choice of $\left\{X_{x}, i_{X}\right\}$ is changed by $\left\{Y_{x}, j_{X}\right\}$, we know (see the end of $\S 2$ ) that the two following elements of $\pi_{1}(\mathbb{G})$,

$$
b_{x, y, z}=\gamma_{X_{x y z}}^{-1}\left(i_{X_{x} \otimes\left(X_{y} \otimes X_{z}\right)} \cdot a_{X_{x}, X_{y}, X_{z}} \cdot i_{\left(X_{x} \otimes X_{y}\right) \otimes X_{z}}^{-1}\right)
$$

and

$$
\bar{b}_{x, y, z}=\gamma_{Y_{x y z}}^{-1}\left(j_{Y_{x} \otimes\left(Y_{y} \otimes Y_{z}\right)} \cdot a_{Y_{x}, Y_{y}, Y_{z}} \cdot j_{\left(Y_{x} \otimes Y_{y}\right) \otimes Y_{z}}^{-1}\right)
$$


determine cohomologous 3 -cocycles of $\pi_{0}(\mathbb{G})$ with con coefficients in $\pi_{1}(\mathbb{G})$. The first choice gives $k^{\varphi, \psi}$ satisfying

$$
\mu_{x, y} T_{z} \cdot \mu_{x y, z}=T_{x} \mu_{y, z} \cdot \mu_{x, y z} \cdot T\left(a_{X_{x}, X_{y}, X_{z}}\right) \cdot k_{x, y, z}^{\varphi, \psi} T_{(x y) z},
$$

and the second choice gives $\bar{k}^{\varphi, \psi}$ satisfying

$$
\mu_{x, y} T_{z} \cdot \mu_{x y, z}=T_{x} \mu_{y, z} \cdot \mu_{x, y z} \cdot \bar{T}\left(a_{Y_{x}, Y_{y}, Y_{z}}\right) \cdot \bar{k}_{x, y, z}^{\varphi, \psi} T_{(x y) z} y,
$$

where $T\left(a_{X_{x}, X_{y}, X_{z}}\right)=\psi\left(b_{x, y, z}\right) T_{(x y) z}$ and $\bar{T}\left(a_{Y_{x}, Y_{y}, Y_{z}}\right)=\psi\left(\bar{b}_{x, y, z}\right) T_{(x y) z}$. Then, comparison and Lemma 4.1 give $\psi\left(b_{x, y, z}\right) \cdot k_{x, y, z}^{\varphi, \psi}=\psi\left(\bar{b}_{x, y, z}\right) \cdot \bar{k}_{x, y, z}^{\varphi, \psi}$. Now, since $\psi$ is a homomorphism of $\pi_{0}(\mathbb{G})$-modules, $\psi\left(b_{x, y, z}\right)$ and $\psi\left(\bar{b}_{x, y, z}\right)$ are cohomologous 3 -cocycles of $\pi_{0}(\mathbb{G})$ with coefficients in the $\pi_{0}(\mathbb{G})$-module, via $\varphi, \pi_{1}(\mathcal{E} q(\mathbb{H}))$ and therefore it is clear that $k^{\varphi, \psi}$ and $\bar{k}^{\varphi, \psi}$ are also cohomologous.

ii) By Lemma 4.1, any other choice $\theta_{x, y}: T_{x y} \rightarrow T_{x} T_{y}$ has the form $\theta_{x, y}=$ $\mu_{x, y} \cdot h_{x, y} T_{x y}$ where $h: \pi_{0}(\mathbb{G})^{2} \rightarrow \pi_{1}(\mathcal{E} q(\mathbb{H}))$ is a normalized 2-cochain. Then, for any objects $X, Y, Z \in \mathbb{G}$, we obtain the following expressions of the isomorphism $J: T_{x y z} \rightarrow T_{x} T_{y} T_{z}$ given by:

$$
J=T_{x} \theta_{x, y} \cdot \theta_{x, y z} \cdot T\left(a_{X_{x}, X_{y}, X_{z}}\right) \cdot k_{x, y, z}^{\prime} T_{(x y) z} \cdot
$$

On the one hand,

$$
\begin{aligned}
& J=T_{x} \mu_{y, z} \cdot T_{x} h_{y, z} T_{y z} \cdot \mu_{x, y z} \cdot h_{x, y z} T_{x(y z)} \cdot T\left(a_{X_{x}, X_{y}, X_{z}}\right) \cdot k_{x, y, z}^{\prime} T_{(x y) z} \\
& \stackrel{(23)}{=} T_{x} \mu_{y, z} \cdot T_{x} h_{y, z} T_{y z} \cdot \mu_{x, y z} \cdot T\left(a_{X_{x}, X_{y}, X_{z}}\right) \cdot h_{x, y z} T_{(x y) z} \cdot k_{x, y, z}^{\prime} T_{(x y) z} \\
& \stackrel{(24)}{=} T_{x} \mu_{y, z} \cdot{ }^{x} h_{y, z} T_{x} T_{y z} \cdot \mu_{x, y z} \cdot T\left(a_{X_{x}, X_{y}, X_{z}}\right) \cdot\left(h_{x, y z} \cdot k_{x, y, z}^{\prime}\right) T_{(x y) z} \\
& \stackrel{(23)}{=} T_{x} \mu_{y, z} \cdot \mu_{x, y z} \cdot{ }^{x} h_{y, z} T_{x(y z)} \cdot T\left(a_{X_{x}, X_{y}, X_{z}}\right) \cdot\left(h_{x, y z} \cdot k_{x, y, z}^{\prime}\right) T_{(x y) z} \\
& \stackrel{(23)}{=} T_{x} \mu_{y, z} \cdot \mu_{x, y z} \cdot T\left(a_{X_{x}, X_{y}, X_{z}}\right) \cdot{ }^{x} h_{y, z} T_{(x y) z} \cdot\left(h_{x, y z} \cdot k_{x, y, z}^{\prime}\right) T_{(x y) z} \\
& =T_{x} \mu_{y, z} \cdot \mu_{x, y z} \cdot T\left(a_{X_{x}, X_{y}, X_{z}}\right) \cdot\left({ }^{x} h_{y, z} \cdot h_{x, y z} \cdot k_{x, y, z}^{\prime}\right) T_{(x y) z}
\end{aligned}
$$

and on the other hand,

$$
\begin{aligned}
& J \stackrel{(29)}{=} \theta_{x, y} T_{z} \cdot \theta_{x y, z} \\
& =\quad \mu_{x, y} T_{z} \cdot h_{x, y} T_{x y} T_{z} \cdot \mu_{x y, z} \cdot h_{x y, z} T_{(x y) z} \\
& \stackrel{(23)}{=} \quad \mu_{x, y} T_{z} \cdot \mu_{x y, z} \cdot h_{x, y} T_{(x y) z} \cdot h_{x y, z} T_{(x y) z} \\
& \stackrel{(29)}{=} T_{x} \mu_{y, z} \cdot \mu_{x, y z} \cdot T\left(a_{X_{x}, X_{y}, X_{z}}\right) \cdot k_{x, y, z} T_{(x y) z} \cdot\left(h_{x, y} \cdot h_{x y, z}\right) T_{(x y) z} \\
& =T_{x} \mu_{y, z} \cdot \mu_{x, y z} \cdot T\left(a_{X_{x}, X_{y}, X_{z}}\right) \cdot\left(k_{x, y, z} \cdot h_{x, y} \cdot h_{x y, z}\right) T_{(x y) z} .
\end{aligned}
$$

Comparison and Lemma 4.1 give

$$
{ }^{x} h_{y, z} \cdot h_{x, y z} \cdot k_{x, y, z}^{\prime}=k_{x, y, z} \cdot h_{x, y} \cdot h_{x y, z},
$$

an equality which asserts that the 3 -cocycles $k$ and $k^{\prime}$ are cohomologous.

iii) If $F_{x} \in \varphi(x), x=[X] \in \pi_{0}(\mathbb{G})$, is another selection of equivalences, then we can select isomorphisms $\epsilon_{x}: T_{x} \rightarrow F_{x}$ and choose, for any $x=[X], y=[Y] \in \pi_{0}(\mathbb{G})$, $\theta_{x, y}: F_{x y} \rightarrow F_{x} F_{y}$ the isomorphism making the following diagram commutative: 


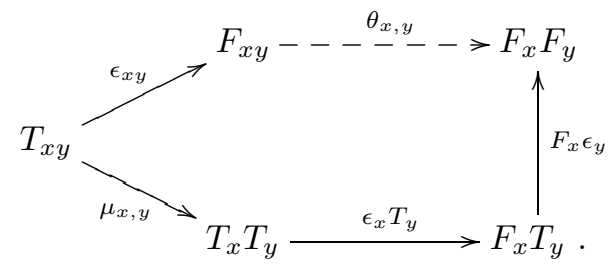

Thus we have,

$$
\begin{aligned}
& \theta_{x, y} F_{z} \cdot \theta_{x y, z} \cdot \epsilon_{(x y) z}=\theta_{x, y} F_{z} \cdot F_{x y} \epsilon_{z} \cdot \epsilon_{x y} T_{z} \cdot \mu_{x y, z} \\
& \stackrel{\text { nat }}{=} \quad F_{x} F_{y} \epsilon_{z} \cdot \theta_{x, y} T_{z} \cdot \epsilon_{x y} T_{z} \cdot \mu_{x y, z} \\
& =F_{x} F_{y} \epsilon_{z} \cdot F_{x} \epsilon_{y} T_{z} \cdot \epsilon_{x} T_{y} T_{z} \cdot \mu_{x, y} T_{z} \cdot \mu_{x y, z} \\
& \stackrel{(29)}{=} \quad F_{x} F_{y} \epsilon_{z} \cdot F_{x} \epsilon_{y} T_{z} \cdot \epsilon_{x} T_{y} T_{z} \cdot T_{x} \mu_{y, z} \cdot \mu_{x, y z} \cdot T\left(a_{X_{x}, X_{y}, X_{z}}\right) \text {. } \\
& k_{x, y, z} T_{(x y) z} \\
& \stackrel{\text { nat }}{=} F_{x} F_{y} \epsilon_{z} \cdot F_{x} \epsilon_{y} T_{z} \cdot F_{x} \mu_{y, z} \cdot \epsilon_{x} T_{y z} \cdot \mu_{x, y z} \cdot T\left(a_{X_{x}, X_{y}, X_{z}}\right) \text {. } \\
& k_{x, y, z} T_{(x y) z} \\
& =F_{x} \theta_{y, z} \cdot F_{x} \epsilon_{y z} \cdot \epsilon_{x} T_{y z} \cdot \mu_{x, y z} \cdot T\left(a_{X_{x}, X_{y}, X_{z}}\right) \cdot k_{x, y, z} T_{(x y) z} \\
& =F_{x} \theta_{y, z} \cdot \theta_{x, y z} \cdot \epsilon_{x(y z)} \cdot T\left(a_{X_{x}, X_{y}, X_{z}}\right) \cdot k_{x, y, z} T_{(x y) z} \\
& \stackrel{(23)}{=} \quad F_{x} \theta_{y, z} \cdot \theta_{x, y z} \cdot F\left(a_{X_{x}, X_{y}, X_{z}}\right) \cdot \epsilon_{(x y) z} \cdot k_{x, y, z} T_{(x y) z} \\
& \stackrel{(23)}{=} \quad F_{x} \theta_{y, z} \cdot \theta_{x, y z} \cdot F\left(a_{X_{x}, X_{y}, X_{z}}\right) \cdot k_{x, y, z} F_{(x y) z} \cdot \epsilon_{(x y) z} \cdot
\end{aligned}
$$

Therefore $\theta_{x, y} F_{z} \cdot \theta_{x y, z}=F_{x} \theta_{y, z} \cdot \theta_{x, y z} \cdot F\left(a_{X_{x}, X_{y}, X_{z}}\right) \cdot k_{x, y, z} F_{(x y) z}$ and the 3 -cocycle $k$ is unchanged.

If $\mathbb{G}$ and $\mathbb{H}$ are categorical groups and $\operatorname{Char}(\mathbb{G}, \mathbb{H})$ denotes the set of collective characters of $\mathbb{G}$ in $\mathbb{H}$, there is a diagram of maps

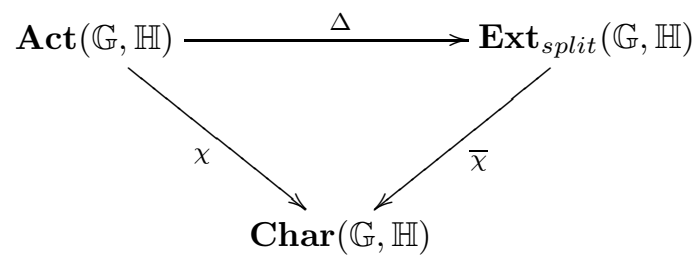

where $\chi$ carries the class of an action of $\mathbb{G}$ on $\mathbb{H}, \mathbf{T}=(T, \mu): \mathbb{G} \rightarrow \mathcal{E} q(\mathbb{H})$, to the collective character $(\varphi, \psi)$ where $\varphi: \pi_{0}(T): \pi_{0}(\mathbb{G}) \rightarrow \pi_{0}(\mathcal{E} q(\mathbb{H}))$ and $\psi=$ $\pi_{1}(T): \pi_{1}(\mathbb{G}) \rightarrow \pi_{1}(\mathcal{E} q(\mathbb{H})$ ) (see last paragraph in $\S 2$ ), $\Delta$ is the bijection (22) and $\bar{\chi}=\chi \Delta^{-1}$. Therefore, $\bar{\chi}: \mathbf{E x t}_{\text {split }}(\mathbb{G}, \mathbb{H}) \rightarrow \operatorname{Char}(\mathbb{G}, \mathbb{H})$ associates a collective character with each equivalence class of split extensions of $\mathbb{G}$ by $\mathbb{H}$. We refer to a collective character $(\varphi, \psi)$ of $\mathbb{G}$ in $\mathbb{H}$ as realizable if it is in the image of $\bar{\chi}$, that is, if it is induced, as explained above, from a split extension of $\mathbb{G}$ by $\mathbb{H}$. The map $\bar{\chi}$ produces a partition of the set of equivalence classes of split extensions

$$
\mathbf{E x t}_{\text {split }}(\mathbb{G}, \mathbb{H})=\coprod_{(\varphi, \psi)} \mathbf{E x t}_{\text {split }}^{(\varphi, \psi)}(\mathbb{G}, \mathbb{H})
$$

where, for any collective character $(\varphi, \psi) \in \mathbf{C h a r}(\mathbb{G}, \mathbb{H}), \mathbf{E x t}_{\text {split }}^{(\varphi, \psi)}(\mathbb{G}, \mathbb{H})=\bar{\chi}^{-1}(\varphi, \psi)$ denotes the fiber over $(\varphi, \psi)$ of $\bar{\chi}$. Hence, a collective character $(\varphi, \psi)$ is realizable 
if the set $\mathbf{E x t}_{\text {split }}^{(\varphi, \psi)}(\mathbb{G}, \mathbb{H})$ is non empty. We refer to $\operatorname{Ext}_{\text {split }}^{(\varphi, \psi)}(\mathbb{G}, \mathbb{H})$ as the set of equivalence classes of realizations of the collective character $(\varphi, \psi)$.

Analogously, let $\operatorname{Act}_{(\varphi, \psi)}(\mathbb{G}, \mathbb{H})=\chi^{-1}(\varphi, \psi)$ be the fiber of map $\chi$ over a collective character $(\varphi, \psi)$ of $\mathbb{G}$ in $\mathbb{H}$. Thus we have the partition

$$
\operatorname{Act}(\mathbb{G}, \mathbb{H})=\coprod_{(\varphi, \psi)} \operatorname{Act}_{(\varphi, \psi)}(\mathbb{G}, \mathbb{H}),
$$

and for any collective character $(\varphi, \psi)$ the bijection

$$
\operatorname{Act}_{(\varphi, \psi)}(\mathbb{G}, \mathbb{H}) \cong \operatorname{Ext}_{s p l i t}^{(\varphi, \psi)}(\mathbb{G}, \mathbb{H}) .
$$

Then we have:

Theorem 4.3. A collective character $(\varphi, \psi)$ of a categorical group $\mathbb{G}$ in a categorical group $\mathbb{H}$ is realizable if and only if its obstruction $k(\varphi, \psi)=\left[k^{\varphi, \psi}\right] \in$ $H_{\varphi}^{3}\left(\pi_{0}(\mathbb{G}), \pi_{1}(\mathcal{E} q(\mathbb{H}))\right)$ vanishes.

Proof. If $(\varphi, \psi)$ is a realizable collective character, then $\operatorname{Act}_{(\varphi, \psi)}(\mathbb{G}, \mathbb{H}) \neq \emptyset$ or, equivalently, there exists an action $\mathbf{T}=(T, \mu): \mathbb{G} \rightarrow \mathcal{E} q(\mathbb{H})$ with $\varphi=\pi_{0}(T)$ y $\psi=$ $\pi_{1}(T)$. Then, in the construction of the 3 -cocycle $k^{\varphi, \psi}$ of $\pi_{0}(\mathbb{G})$ with coefficients in the $\pi_{0}(\mathbb{G})$-module, via $\varphi, \pi_{1}(\mathcal{E} q(\mathbb{H}))$ one can take just the equivalences $T_{x}: \mathbb{H} \rightarrow \mathbb{H}$, $x \in \pi_{0}(\mathbb{G})$, given by $T_{x}=T(X)$ and the isomorphisms $\mu_{x, y}$ those given by the homomorphism $\mathbf{T}=(T, \mu)$, that is, $\mu_{x, y}=\mu_{X_{x}, X_{y}}$. In this case, since in $\mathcal{E} q(\mathbb{H})$ the associativity is strict, the condition of $T$ being a homomorphism (3) reduces to the following equality:

$$
\mu_{x, y} T_{z} \cdot \mu_{x y, z}=T_{x} \mu_{y, z} \cdot \mu_{x, y z} \cdot T\left(a_{X_{x}, X_{y}, X_{z}}\right) .
$$

Thus, $k_{x, y, z}^{\varphi, \psi}=1$ according to $(29)$ and so $k(\varphi, \psi)=\left[k^{\varphi, \psi}\right]$ is the zero cohomology class in $H_{\varphi}^{3}\left(\pi_{0}(\mathbb{G}), \pi_{1}(\mathcal{E} q(\mathbb{H}))\right)$.

Conversely, suppose that $(\varphi, \psi)$ is a collective character such that $k(\varphi, \psi)$ is the zero class. Once we have selected equivalences $T_{x}: \mathbb{H} \rightarrow \mathbb{H}, x \in \pi_{0}(\mathbb{G})$, with $T_{1}=i d_{\mathbb{H}}$, Proposition 4.2, ii), assures that there is a choice of isomorphisms $\mu_{x, y}$ : $T_{x y} \rightarrow T_{x} T_{y}$ with $\mu_{1, y}=i d=\mu_{x, 1}$ such that $k^{\varphi, \psi}$ is identically 1 . This means that equality (29) becomes:

$$
\mu_{x, y} T_{z} \cdot \mu_{x y, z}=T_{x} \mu_{y, z} \cdot \mu_{x, y z} \cdot T\left(a_{X_{x}, X_{y}, X_{z}}\right),
$$

and so, according to (3) and (28), $\mathbf{T}: \mathbb{G} \rightarrow \mathcal{E} q(\mathbb{H})$ is a homomorphism of categorical groups with $\chi(T, \mu)=(\varphi, \psi)$. Therefore $\operatorname{Act}_{(\varphi, \psi)}(\mathbb{G}, \mathbb{H}) \cong \mathbf{E x t}_{\text {split }}^{(\varphi, \psi)}(\mathbb{G}, \mathbb{H})$ is not empty so that the collective character $(\varphi, \psi)$ is realizable.

To complete the classification of the split extensions of categorical groups, we reach the result below.

Theorem 4.4. If a collective character $(\varphi, \psi)$ is realizable, then the set $\mathbf{E x t}_{\text {split }}^{(\varphi, \psi)}(\mathbb{G}, \mathbb{H})$ of equivalence clases of realizations of $(\varphi, \psi)$ is a principal homogeneous spade under the abelian group $H_{\varphi}^{2}\left(\pi_{0}(\mathbb{G}), \pi_{1}(\mathcal{E} q(\mathbb{H}))\right)$. In particular, there is a (non-natural) bijection

$$
\operatorname{Ext}_{\text {split }}^{(\varphi, \psi)}(\mathbb{G}, \mathbb{H}) \cong H_{\varphi}^{2}\left(\pi_{0}(\mathbb{G}), \pi_{1}(\mathcal{E} q(\mathbb{H}))\right) .
$$


Proof. We describe an action

$$
H_{\varphi}^{2}\left(\pi_{0}(\mathbb{G}), \pi_{1}(\mathcal{E} q(\mathbb{H}))\right) \times \operatorname{Act}_{(\varphi, \psi)}(\mathbb{G}, \mathbb{H}) \longrightarrow \operatorname{Act}_{(\varphi, \psi)}(\mathbb{G}, \mathbb{H})
$$

set out below.

Let $h: \pi_{0}(\mathbb{G})^{2} \rightarrow \pi_{1}(\mathcal{E} q(\mathbb{H}))$ be a normalized 2-cocycle representative of an element $[h] \in H_{\varphi}^{2}\left(\pi_{0}(\mathbb{G}), \pi_{1}(\mathcal{E} q(\mathbb{H}))\right)$ and $(T, \mu)$ a representative of an element $[T, \mu] \in \operatorname{Act}_{(\varphi, \psi)}(\mathbb{G}, \mathbb{H})$. Then, if for each pair of objects $X, Y \in \mathbb{G}$ we consider $x=[X], y=[Y]$ in $\pi_{0}(\mathbb{G})$ and the isomorphism $(h+\mu)_{x, y}: T_{x y} \rightarrow T_{x} T_{y}$ defined by $(h+\mu)_{x, y}=h_{x, y} T_{x} T_{y} \cdot \mu_{x, y}$ we observe, simply by reversing the proof of Proposition 4.2 , ii), that we have a new action $(T, h+\mu)$, representing another element $[T, h+\mu] \in \operatorname{Act}_{(\varphi, \psi)}(\mathbb{G}, \mathbb{H})$, which we maintain depends only on $[h]$ and $[T, \mu]$. To see this, let us suppose that $g$ is another representative of $[h]$ and $(F, \theta)$ is another representative of $[T, \mu]$. There must then exist isomorphisms $\psi_{x}: i d_{\mathbb{H}} \rightarrow i d_{\mathbb{H}}$ and $\epsilon_{x}: T_{x} \rightarrow F_{x}, x \in \pi_{0}(\mathbb{G})$, such that, for any $x, y \in \pi_{0}(\mathbb{G})$, the following equalities hold:

$$
\begin{gathered}
{ }^{x} \psi_{y} \cdot \psi_{x} \cdot h_{x, y}=g_{x, y} \cdot \psi_{x y}, \\
F_{x} \epsilon_{y} \cdot \epsilon_{x} T_{y} \cdot \mu_{x, y}=\theta_{x, y} \cdot \epsilon_{x y} .
\end{gathered}
$$

Then, if we consider the isomorphisms $\left.(\psi+\epsilon)_{x}\right)=\psi_{x} F_{x} \cdot \epsilon_{x}: T_{x} \rightarrow F_{x}$, we have that the actions $(T, h+\mu)$ and $(F, g+\theta)$ are equivalent since, for any $X, Y, Z \in \mathbb{G}$, if $x=[X], y=[Y]$ and $z=[Z]$ are their classes in $\pi_{0}(\mathbb{G})$, we have:

$$
\begin{aligned}
& F_{x}(\psi+\epsilon)_{y} \cdot(\psi+\epsilon)_{x} T_{y} \cdot(h+\mu)_{x, y}=F_{x} \psi_{y} F_{y} \cdot F_{x} \epsilon_{y} \cdot \psi_{x} F_{x} T_{y} . \\
& \epsilon_{x} T_{y} \cdot h_{x, y} T_{x} T_{y} \cdot \mu_{x, y} \\
& \stackrel{(23)}{=} F_{x} \psi_{y} F_{y} \cdot \psi_{x} F_{x} F_{y} \cdot F_{x} \epsilon_{y} \cdot \epsilon_{x} T_{y} \text {. } \\
& \mu_{x, y} \cdot h_{x, y} T_{x y} \\
& \stackrel{(32)}{=} F_{x} \psi_{y} F_{y} \cdot \psi_{x} F_{x} F_{y} \cdot \theta_{x, y} \cdot \epsilon_{x y} \cdot h_{x, y} T_{x y} \\
& \stackrel{(23)}{=} F_{x} \psi_{y} F_{y} \cdot \psi_{x} F_{x} F_{y} \cdot \theta_{x, y} \cdot h_{x, y} F_{x y} \cdot \epsilon_{x y} \\
& \stackrel{(23)}{=} F_{x} \psi_{y} F_{y} \cdot \psi_{x} F_{x} F_{y} \cdot h_{x, y} F_{x} F_{y} \cdot \theta_{x, y} \cdot \epsilon_{x y} \\
& \stackrel{(24)}{=}{ }^{x} \psi_{y} F_{x} F_{y} \cdot \psi_{x} F_{x} F_{y} \cdot h_{x, y} F_{x} F_{y} \cdot \theta_{x, y} \cdot \epsilon_{x y} \\
& \stackrel{(31)}{=} g_{x, y} F_{x} F_{y} \cdot \psi_{x y} F_{x} F_{y} \cdot \theta_{x, y} \cdot \epsilon_{x y} \\
& \stackrel{(23)}{=} g_{x, y} F_{x} F_{y} \cdot \theta_{x, y} \cdot \psi_{x y} F_{x y} \epsilon_{x y} \\
& =(g+\theta)_{x, y} \cdot(\psi+\epsilon)_{x y} \text {. }
\end{aligned}
$$

Therefore, $([h],[T, \mu]) \mapsto[h]+[T, \mu]=[T, h+\mu]$ is a well-defined action of the abelian group $H_{\varphi}^{2}\left(\pi_{0}(\mathbb{G}), \pi_{1}(\mathcal{E} q(\mathbb{H}))\right)$ on $\operatorname{Act}_{(\varphi, \psi)}(\mathbb{G}, \mathbb{H})$.

The above action is principal since, if we suppose that $[T, h+\mu]=[T, \mu]$, there must exist a family of isomorphisms $\epsilon_{x}: T_{x} \rightarrow T_{x}$ such that, for any $x, y \in \pi_{0}(\mathbb{G})$, $T_{x} \epsilon_{y} \cdot \epsilon_{x} T_{y} \cdot(h+\mu)_{x, y}=\mu_{x, y} \cdot \epsilon_{x y}$. Then, by Lemma 4.1, we can write $\epsilon_{x}=u_{x} T_{x}$ for a uniquely determined cochain $u: \pi_{0}(\mathbb{G}) \rightarrow \pi_{1}(\mathcal{E} q(\mathbb{H}))$ and we have 


$$
\begin{aligned}
& \mu_{x, y} \cdot u_{x y} T_{x y}=\mu_{x, y} \cdot \epsilon_{x y} \\
& =T_{x} \epsilon_{y} \cdot \epsilon_{x} T_{y} \cdot h_{x, y} T_{x} T_{y} \cdot \mu_{x, y} \\
& =T_{x} u_{y} T_{y} \cdot u_{x} T_{x} T_{y} \cdot h_{x, y} T_{x} T_{y} \cdot \mu_{x, y} \\
& \stackrel{(24)}{=} \quad{ }^{x} u_{y} T_{x} T_{y} \cdot u_{x} T_{x} T_{y} \cdot h_{x, y} T_{x} T_{y} \cdot \mu_{x, y} \\
& =\left({ }^{x} u_{y} \cdot u_{x} \cdot h_{x, y}\right) T_{x} T_{y} \cdot \mu_{x, y} \\
& \stackrel{(23)}{=} \mu_{x, y} \cdot\left({ }^{x} u_{y} \cdot u_{x} \cdot h_{x, y}\right) T_{x y},
\end{aligned}
$$

from which we deduce that $u_{x y}={ }^{x} u_{y} \cdot u_{x} \cdot h_{x, y}$, that is, $h=\partial(u)$ represents the zero class in $H_{\varphi}^{2}\left(\pi_{0}(\mathbb{G}), \pi_{1}(\mathcal{E} q(\mathbb{H}))\right)$.

Finally we observe that the action is transitive. For this, let $(T, \beta),(F, \theta)$ be any two actions representing elements in $\operatorname{Act}_{(\varphi, \psi)}(\mathbb{G}, \mathbb{H})$. For each $X \in \mathbb{G}$, if $x=[X]$ in $\pi_{0}(\mathbb{G})$, we have that $T_{x}, F_{x} \in \varphi(x)$ and then there must exist isomorphisms $\epsilon_{x}: T_{x} \rightarrow F_{x}, x \in \pi_{0}(\mathbb{G})$, with $\epsilon_{1}=i d_{i d_{\mathbb{H}}}$. Then, as in the proof of Proposition 4.2 , iii), by choosing $\mu_{x, y}: T_{x y} \rightarrow T_{x} T_{y}$ the isomorphisms such that $\mu_{x, y} \cdot \epsilon_{x y}=$ $T_{x} \epsilon_{y} \cdot \epsilon_{x} T_{y} \cdot \theta_{x, y}, x, y \in \pi_{0}(\mathbb{G})$, we find a new action $(T, \mu)$ that represents the same class in $\operatorname{Act}_{(\varphi, \psi)}(\mathbb{G}, \mathbb{H})$ as $(F, \theta)$. Now, by Lemma 4.1, we can write

$$
\beta_{x, y}=h_{x, y} T_{x} T_{y} \cdot \mu_{x, y}
$$

where $h: \pi_{0}(\mathbb{G})^{2} \rightarrow \pi_{1}(\mathcal{E} q(\mathbb{H}))$ is a normalized 2-cochain of $\pi_{0}(\mathbb{G})$ with coefficients in $\pi_{1}(\mathcal{E} q(\mathbb{H}))$, and, just as in the proof of Proposition 4.2 ii), (for $k=k^{\prime}=1$ ), we see that ${ }^{x} h_{y, z} \cdot h_{x, y z}=h_{x, y} \cdot h_{x y, z}$. Hence, $h$ is a 2-cocycle of $\pi_{0}(\mathbb{G})$ with coefficients in $\pi_{1}(\mathcal{E} q(\mathbb{H}))$ and clearly, according to $(33)$, we have $(T, h+\mu)=(T, \beta)$ and so $[h]+[F, \theta]=[h]+[T, \mu]=[T, h+\mu]=[T, \beta]$ and the action is transitive.

\section{References}

[1] R. Baer, Erweiterung gruppen und ihren isomorphismen, Math. Z. 38 (1935), $375-416$.

[2] F. Borceux, Handbook of categorical algebra 1,2, Cambridge University Press (1994).

[3] L. Breen, Théorie de Schreier supérieure, Ann. scient. Éc. Norm. Sup. $4^{e}$ série, 25 (1992), 465-514.

[4] R. Brown and C.B. Spencer. G-groupoids, crossed modules and the fundamental groupoid of a topological group. Proc. Kon. Ned. Acad. v. Wet. 79 (1976) 296-302.

[5] P. Carrasco and A.M. Cegarra, (Braided) Tensor structures on homotopy groupoids and nerves of (braided) categorical groups, Communications in Algebra 24 (13) (1996), 3995-4058.

[6] P. Carrasco and A.M. Cegarra, Schreier theory for central extensions of categorical groups, Communications in Algebra 24 (13) (1996), 4059-4112.

[7] P. Carrasco, A.R. Garzón and J.G. Miranda, Schreier theory for singular extensions of categorical groups and homotopy classification, Communications in Algebra 28(5) (2000), 2585-2613.

[8] A.M. Cegarra, M. Bullejos, A.R. Garzón, Higher dimensional obstruction theory in algebraic categories, J. Pure Appl. Algebra 49 (1987), 43-102. 
[9] P. Dedecker, Cohomologie non-abélienne, Séminaire de l'Institut Mathématique Lille (1965).

[10] J. Duskin, Non-abelian monadic cohomology and low-dimensional obstruction theory, Math. Forschung Inst. Oberwolfach Tagungsberich 33 (1976).

[11] S. Eilenberg and S. Mac Lane, Cohomology theory in Abstract group II, Ann. of Math. 48 (1946) 326-341.

[12] A.R. Garzón, J.G. Miranda and A. del Río, Tensor structures on homotopy groupoids of topological spaces, submitted.

[13] A. Joyal and R. Street, Braided tensor categories, Advances in Math. (1) 82 (1991), 20-78.

[14] G. M. Kelly, On Mac Lane's conditions for coherence of natural associativities, commutativities, etc. J. of Algebra, 1 (1964), 397-402.

[15] S. Mac Lane, Natural associativity and commutativity, Rice University Studies 49 (1963), 28-46.

[16] K.J. Norrie, Actions and automorphisms of crossed modules Bull. Soc. Math. France 118 (1990), 129-146.

[17] N. Saavedra, Catégories tannakiennes, Lecture Notes in Math. 265, Springer Verlag (1972).

[18] O. Schreier, Über die Erweiterung von Gruppen I, Monatsh. Math. Phys. 34 (1926), 165-180.

[19] H.X. Sinh, Gr-catégories, (Université Paris VII, Thése de doctorat, 1975).

[20] O. Teichmüller, Über die sogenannte nichtkommutative Galoissche Theorie und die Relation $\xi_{\lambda, \mu, \nu} \xi_{\lambda, \mu \nu, \pi} \xi_{\mu, \nu, \pi}^{\lambda}=\xi_{\lambda, \mu, \nu \pi} \xi_{\lambda \mu, \nu, \pi}$, Deutsche Math, 5 (1940) 138-149.

[21] K.-H. Ulbrich, Group Cohomology for Picard Categories. J. of Algebra 91 (1984), 464-498.

This article may be accessed via WWW at http://www.rmi.acnet.ge/hha/ or by anonymous ftp at

ftp://ftp.rmi.acnet.ge/pub/hha/volumes/2001/n6/n6.(dvi,ps,dvi.gz,ps.gz)

Antonio R. Garzón agarzon@ugr.es

Departamento de Álgebra

Facultad de Ciencias

Universidad de Granada

18071 Granada

Spain.

Hvedri Inassaridze hvedri@rmi.acnet.ge

A. Razmadze Mathematical Institute

Georgian Academy of Sciences

1, M. Aleksidze St., Tbilisi 380093

Georgia. 